\title{
A Hybrid Method for Modeling and Solving Supply Chain Optimization Problems with Soft and Logical Constraints
}

\author{
Paweł Sitek, ${ }^{1}$ Krzysztof Bzdyra, ${ }^{2}$ and Jarosław Wikarek ${ }^{1}$ \\ ${ }^{1}$ Institute of Management and Control Systems, Kielce University of Technology, Kielce, Poland \\ ${ }^{2}$ Department of Computer Science and Management, Koszalin University of Technology, Kielce, Poland \\ Correspondence should be addressed to Krzysztof Bzdyra; krzysztof.bzdyra@tu.koszalin.pl
}

Received 3 February 2016; Accepted 7 August 2016

Academic Editor: Jacques Ferland

Copyright (c) 2016 Paweł Sitek et al. This is an open access article distributed under the Creative Commons Attribution License, which permits unrestricted use, distribution, and reproduction in any medium, provided the original work is properly cited.

\begin{abstract}
This paper presents a hybrid method for modeling and solving supply chain optimization problems with soft, hard, and logical constraints. Ability to implement soft and logical constraints is a very important functionality for supply chain optimization models. Such constraints are particularly useful for modeling problems resulting from commercial agreements, contracts, competition, technology, safety, and environmental conditions. Two programming and solving environments, mathematical programming (MP) and constraint logic programming (CLP), were combined in the hybrid method. This integration, hybridization, and the adequate multidimensional transformation of the problem (as a presolving method) helped to substantially reduce the search space of combinatorial models for supply chain optimization problems. The operation research MP and declarative CLP, where constraints are modeled in different ways and different solving procedures are implemented, were linked together to use the strengths of both. This approach is particularly important for the decision and combinatorial optimization models with the objective function and constraints, there are many decision variables, and these are summed (common in manufacturing, supply chain management, project management, and logistic problems). The ECL ${ }^{\mathrm{i}} \mathrm{SS}^{\mathrm{e}}$ system with Eplex library was proposed to implement a hybrid method. Additionally, the proposed hybrid transformed model is compared with the MILP-Mixed Integer Linear Programming model on the same data instances. For illustrative models, its use allowed finding optimal solutions eight to one hundred times faster and reducing the size of the combinatorial problem to a significant extent.
\end{abstract}

\section{Introduction}

A supply chain (SC) is a system of producers, distributors, suppliers networks, customers, retailers, activities, resources, and information involved in moving services and/or products from producers to customers. All members of the SC are working together to achieve this goal [1]. Supply chain management (SCM) covers planning, control, and management of all activities and resources in production, distribution, and transportation.

Simultaneous consideration of distribution, production, and transport planning and control problems greatly advances the effectiveness of the processes and operations of all of these problems. These problems are characterized by high complexity due to the large number of different activities of the SC (plants, vehicles, distribution centers, retailers, etc.) and due to many interactions and different restrictions among these activities (i.e., capacity, modes of transportation, relocation of distribution centers, nature of demand, balance, environmental, etc.). For modeling these restrictions it is usually sufficient to use linear, integer, or binary constraints.

In contrast, soft and logical constraints are better suited to modeling issues related to trade agreements, marketing, contracts, competition, security, finance, and so forth.

Thus, the nature of the SC problems is characterized by a number of constraints and discrete decision variables, as confirmed by a variety of optimization models, the review of which is presented in $[2,3]$. The structure of decision and optimization problems in a SC poses a real challenge in terms of mathematical programming (MP) application to both their modeling and their optimization. Very often discussed problems become overconstrained (the problem where solution does not exist, i.e., valuation of variables that satisfies all the constraints). 
For this reason, the main motivation behind this study was to develop an alternative approach, highly effective in optimization and far more flexible in problem modeling than mathematical programming methods, especially when modeling logical and soft constraints.

A contribution of this paper is to propose a declarative method for hybrid modeling and solving of the supply chain optimization problems using multidimensional transformation as a presolving method. Moreover, a hybrid transformed combinatorial model with soft, hard, and logical constraints for supply chain optimization problems has been described. In addition, a declarative hybrid implementation framework has been presented, in which mathematical programming (MP) and constraint logic programming (CLP) are integrated.

A declarative constraint logic programming environment with MP-library (Eplex) as a declarative hybrid framework was chosen for the implementation of this method and model.

The rest of this paper is organized as follows: Section 2 describes material and methods. Section 3 gives the main assumptions of the declarative method with MP-library and describes implementation framework. The optimization models (MILP, hybrid, and hybrid transformed), computational examples, and efficiency tests of the declarative implementation framework are shown in Section 4. The summary and discussion on possible extensions are included in Section 5.

\section{Methods}

The use of constraint-based methods for the modeling of decision and optimization problems that contain constraints appears to be natural and effective owing to their character and properties. Constraints reflect the logical relationships between decision variables. Each variable can take values within a given domain-constraints restrict the ranges of those values. The properties of constraints include the following:

(i) Declarativity. Define the relationships between variables; no computation algorithm is needed.

(ii) Additivity. The set of constraints, their context, and conjunction are important, not their sequence in adding to each other.

(iii) Dependence. Constraints often share decision variables, commonly in the problems of supply chain management, production, transport, and logistics.

(iv) Domain Solution. Constraints rarely specify the values of decision variables - the domains within which the variables can take on values are specified more often.

These properties and the character of constraints make them a natural form of knowledge and information modeling, particularly adequate in such areas as production, logistics, transport, or distribution, where they become resource, time, environment, transport, or finance constraints of linear, nonlinear, logical, or integer type. An optimization problem of
SCM connects the issues of storage, production, distribution, and transportation taking account of conditions relating to time, environment, and finance.

Therefore, we claim that constraint-based environments are a natural way of modeling the problem with constraints [4-8]. These environments usually use the declarative programming paradigm which specifies the conditions the solution has to satisfy (what is to be achieved) and not the detailed algorithm (how it should be achieved). The constraint satisfaction problem (CSP) is the fundamental concept of constraint-based declarative environments $[4,9]$.

CSP is a mathematical problem described as a set of objects whose condition must satisfy a set of constraints.

Formally, CSP is described by a set of decision variables $V_{1}, V_{2}, \ldots, V_{n}$ and set of constraints $\mathrm{Con}_{1}, \mathrm{Con}_{2}, \ldots, \mathrm{Con}_{m}$. Each decision variable $V_{i}$ has a domain Dom $_{i}$ of possible variables. Each constraint $\mathrm{Con}_{i}$ involves some subset of the decision variables and determines the combination of values for this subset. A state of the CSP is described by an assignment of values to decision variables. An assignment that meets all constraints is named a consistent/legal assignment.

CSPs are usually solved using a different form of search. The most used methods include constraint propagation [6], different variants of backtracking [10], and local search.

These techniques allow one to quickly find a domain solution or conclude that domains are contradictory.

CSPs are the basis for constraint programming (CP)/ constraint logic programming (CLP) environments [4].

Several modifications of the classic model of CSP have been proposed: flexible CSPs (relaxing assumptions that each solution must satisfy all constraints, including FuzzyCSP), dynamic CSPs (a sequence of the static CSPs), and decentralized CSPs [11].

Problem representation as a CSP offers several advantages. First, it is closer to a natural presentation of a problem (constraints over variables). Second, there is no requirement for presenting the solving method/algorithm. Finally, its effectiveness for yes/no decision problems is higher than that of traditional methods.

The use of a declarative method, for example, CLP, offers better decision and optimization modeling possibilities compared to the MP-based approach. Advantages and shortcomings of both (MP and CLP) are widely reported in the literature $[12,13]$ including our own experience $[14,15]$.

In MP environments, the number of modeling constraint types is modest, including only linear and integer constraints. CLP environments make use of a wider range of constraints; in addition to those named above, nonlinear, logical, or predefined symbolic (cumulative, alldifferent, disjunctive, etc.) constraints are embedded. Different types of constraints require different computational effort in both environments. MP-based approaches are effective in solving linear constraints and optimization but poor at handling integer (binary) constraints. Logical constraints are hard to model in the MP-based environments, where a number of linear and integer constraints (common in SCM problems) have to be contained. In CLP-based environments, integer and binary (domain integrity) and logical and nonlinear constraints are easy to model and solve. However, linear 


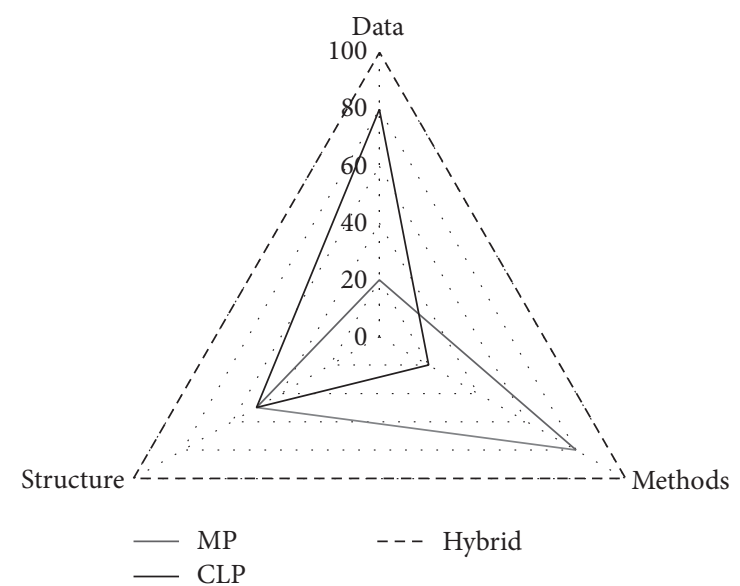

Figure 1: Areas used in CLP and MP and hybrid method.

constraints, which add up many decision variables, increase the computational effort and extend the solving time, especially when an optimization problem is being solved. Yet, this constraint type is common in production, distribution, and transportation problems, thus for SCM optimization.

Therefore, MP-based environments are effective in solving linear constraints and optimization. In contrast, the CPbased environments are effective for modeling and solving integer and logical constrains and finding feasible solutions.

Each issue of problem modeling and solving can be considered in three areas (dimensions). The first concerns the proposed method for both modeling and solving. The second relates to the structure and nature of the problem (types of constraints, the decision or optimization problem, etc.). The last area refers to the data instances. Each of the presented environments uses these areas in a different way (Figure 1). CLP mainly uses the data instances and slightly less the structure of the problem. Due to the declarative nature, external methods or algorithms are not required. The CLP model is already the method. MP mainly focuses on the method and then the structure and properties of the problem. The data instances are outside of MP model. Therefore, hybrid method allows maximum exploration of all these three areas (Figure 1).

The majority of supply chain optimization problems are formulated as MP-based models (MIP-Mixed Integer Programming; MILP, Mixed Integer Linear Programming; IP, Integer Programming; and MOOP, multiobjective optimization programming) $[2,6,7]$.

Due to the properties and character of the models of supply chain optimization and a large number of integer decision variables, MP-based models and methods can only be used to solve problems of small size. Another weak point of these models is that only integer, linear, and binary constraints can be applied. In fact, issues related to the supply chain optimization are often nonlinear, logical, and so forth.

For the above reasons, the problems were formulated in a new way with new hybrid transformed models.
The most important objectives behind this research were to develop hybrid optimization models and methods for supply chain optimization problems using a declarative hybrid method with multidimensional transformation.

Hybridization results from the fact that what is difficult to model and solve in CLP can be easily modeled and solved in MP and vice versa. The best programming framework for the implementation of the hybrid models and methods is a declarative CLP environment with MP-library. Moreover, such declarative hybrid models and methods allow the use of all areas (dimensions) of the problem (methods, structure, and data) to solve it (Figure 1). Finally, this method allows the multidimensional transformation of the problem (Section 4.3), which reduces its size and significantly affects the speed of finding a solution. Among other things, the presented method differs from the integrative approaches $\mathrm{CP} / \mathrm{MP}$ known from the literature $[12,13,16,17]$ in the use of multidimensional transformation as an integral part of the declarative hybrid method.

\section{The Concept and Implementation of the Declarative Hybrid Method}

Based on the characteristics of the CLP and MP environments, their mutual complementarity, the properties of supply chain optimization problems, and previous experiences with hybridization $[14,15]$ we have proposed the concept of a declarative method for modeling and optimization. The main assumptions of the proposed concept are as follows:

(i) The problem is modeled by a set of facts and a set of constraints (integer, linear, logical, etc.).

(ii) Models can be formulated in the form of MIP/MILP/ IP models or hybrid models.

(iii) Constraints may take two forms: soft and hard.

(iv) The problem is modeled by CLP-based predicates, a far more flexible technique than the MP-based procedures and formulas.

(v) Multidimensional transforming of the model as a presolving method is done by CLP-based predicates (Section 3.1).

(vi) Optimization is done through MP-based procedures.

The scheme of the implementation framework for declarative hybrid method is presented in Figure 2.

From a variety of declarative constraint programming environments for the implementation of the framework, ECL ${ }^{i} P S^{e}$ software $[4,18]$ was selected. ECL ${ }^{i} P S^{e}$ is a high-level modeling, control, and declarative programming language with interfaces to third-party solvers. ECL ${ }^{i} \mathrm{PS}^{\mathrm{e}}$ was used to model the problem, transform it, and search for a domain solution by constraint propagation (Figure 2, Table 1). This solution was then the basis for the final MILP model, developed in the Eplex library [19] of the ECL ${ }^{\mathrm{i}} \mathrm{PS}^{\mathrm{e}}$ environment. The Eplex library allows MIP/IP/MILP problems to be modeled in ECL $^{\mathrm{i}} \mathrm{PS}^{\mathrm{e}}$ environment and optimized by an external mathematical programming solver. 


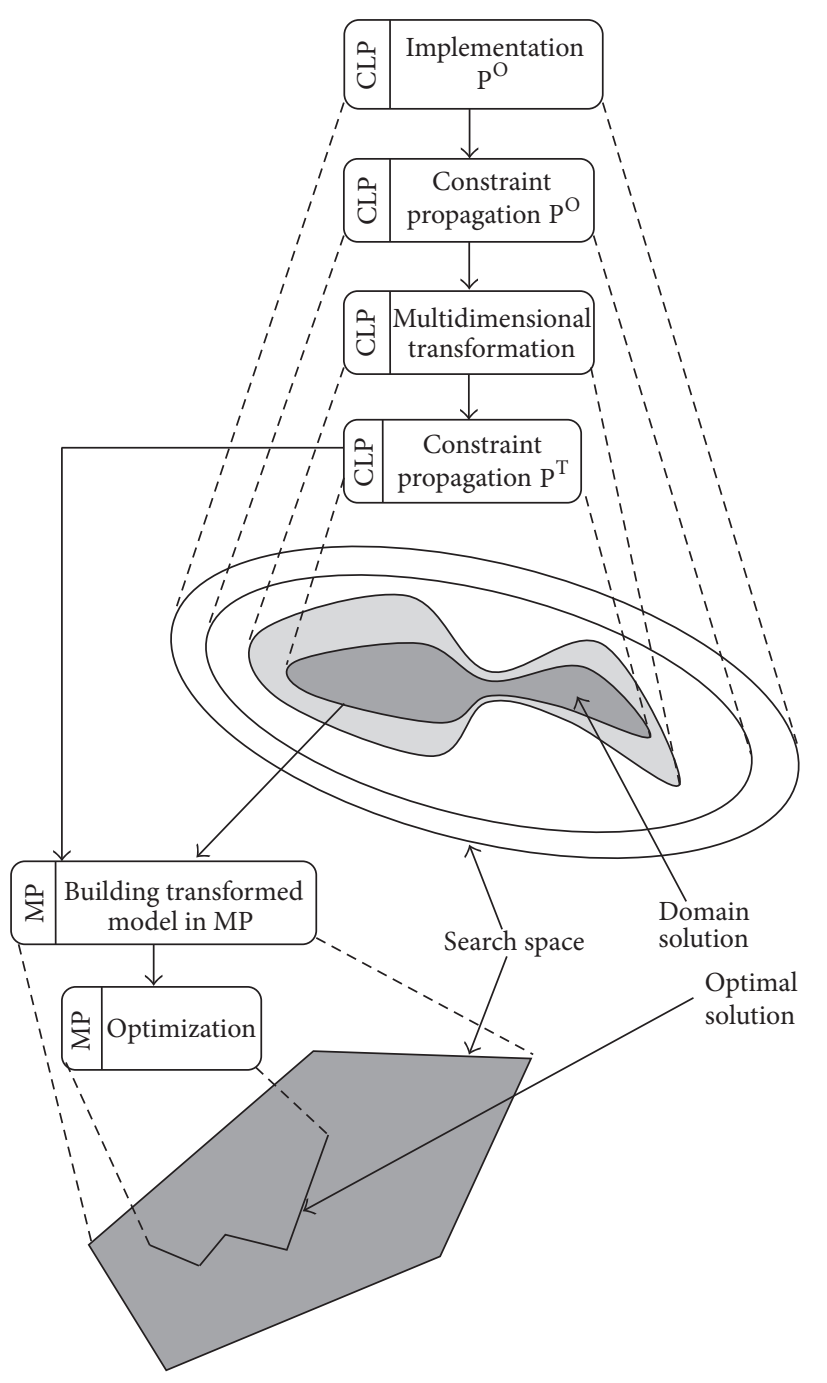

$\mathrm{P}^{\mathrm{O}}$ : original problem $\quad$ CLP: constraint logic programming $\mathrm{P}^{\mathrm{T}}$ : transformed problem $\quad$ MP: mathematical programming

FIgURE 2: The conceptual scheme of the declarative hybrid implementation framework (DHIF).

3.1. The Concept of Multidimensional Transformation of the Problem. Due to the nature of models in SC/SCM (a large number of integer decision variables, summation of many decision variables in the objective function and constraints), the constraint propagation efficiency decreases significantly.

Constraint propagation [4] is an important component of constraint logic programming affecting the efficiency and effectiveness of the search solution. For these reasons, studies into more effective way of constraint propagation have been performed $[14,15]$. The result of this study was to propose a new multidimensional transformation of the problem as a presolving method. Transformation is this element of this hybrid method which distinguishes it from the MP and CLP integration methods reported in the literature [13, 20, 21]. This method proposes a multidimensional transformation of a problem $(1 \mathrm{M})$, which involves decision variables $(2 \mathrm{M})$ and
TABLE 1: Symbols used in the multidimensional transformation of the problem.

\begin{tabular}{ll}
\hline Symbol & Description of the symbol \\
\hline $\mathrm{P}^{\mathrm{O}}$ & Original problem \\
$X^{\mathrm{O}}$ & Decision variables before transformation \\
$\mathrm{C}^{\mathrm{O}}$ & Constraints before transformation \\
$\mathrm{P}^{\mathrm{T}}$ & Transformed problem \\
$X^{\mathrm{T}}$ & Decision variables after transformation \\
$\mathrm{C}^{\mathrm{T}}$ & Constraints after transformation \\
$\mathrm{C}^{\mathrm{AT}}$ & Additional constraints that limit solution search \\
$\mathrm{S}$ & space \\
$\mathrm{D}$ & Structure and properties of the modeled problem \\
& Data (specific instances of data values for the \\
CLP & parameters of the problem) \\
$\mathrm{T}^{\mathrm{CLP}}$ & $\begin{array}{l}\text { Properties and methods of the CLP (constraint } \\
\text { propagation, local search, domain solving, etc.) }\end{array}$ \\
\hline
\end{tabular}

constraints $(3 \mathrm{M})$. The following dimensions are considered $(4 \mathrm{M})$ : data (D), properties and the structure of the problem (S), and the properties of the modeling and solving CLP environment (CLP). Table 1 describes the symbols used in the transformation.

$$
\begin{gathered}
\mathrm{P}^{\mathrm{O}} \stackrel{\mathrm{T}^{\mathrm{CLP}}}{\longrightarrow} \mathrm{P}^{\mathrm{T}}, \\
X^{\mathrm{O}} \stackrel{\mathrm{T}^{\mathrm{CLP}}}{\longrightarrow} X^{\mathrm{T}}, \\
\mathrm{C}^{\mathrm{O}} \stackrel{\mathrm{T}^{\mathrm{CLP}}}{\longrightarrow} \mathrm{C}^{\mathrm{T}}, \mathrm{C}^{\mathrm{AT}}, \\
\mathrm{T}^{\mathrm{CLP}}[\mathrm{S}, \mathrm{D}, \mathrm{CLP}] .
\end{gathered}
$$

The transformation $\mathrm{T}^{\mathrm{CLP}}(4 \mathrm{M})$, performed in the CLP environment, results in the following (Section 4.3):

(i) The reduction in the total number of and changing the nature of decision variables attained through aggregation and elimination.

(ii) The reduction in the number of constraints resulting from the problem properties.

(iii) The introduction of additional constraints $\mathrm{C}^{\mathrm{AT}}$ that limit the solution search space without affecting the problem (Table 6).

(iv) The reduction of the solution search time.

\section{Illustrative Examples}

The proposed method has been verified on two SCM optimization illustrative examples. First example was formulated as MILP model based on [22] overconstraints (2)-(27). This model was used to compare the DHIF relative to the classical MP solver for the same data sets $[22,23]$. Then the hybrid model (HM) (1)-(30) was implemented and solved only in DHIF because the declarative and logical character of 


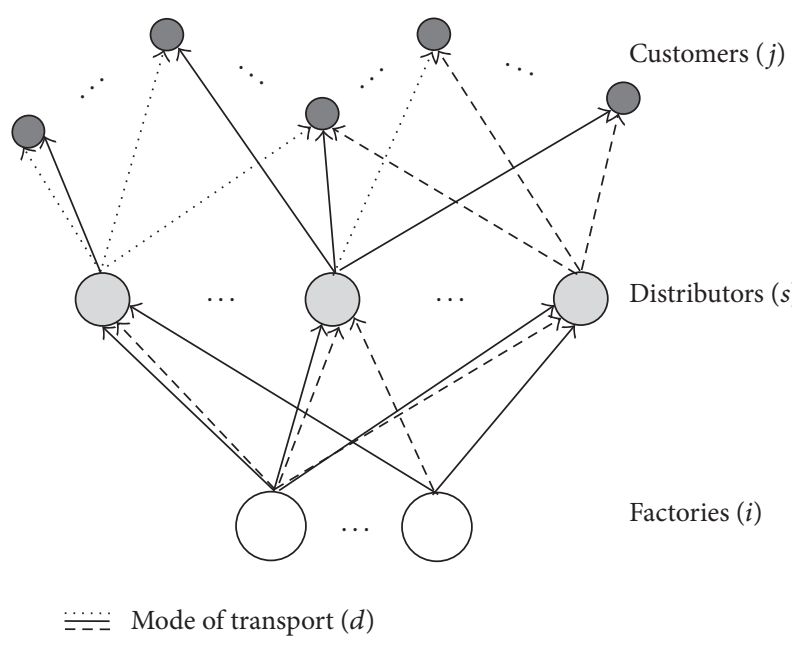

FIGURE 3: The scheme of exemplified structure of the supply chain network with multimodal transport.

constraints (28)-(30) weakens the structure of the model. In addition, these constraints are difficult to implement in the MP-based environment and cause the increase in the number of decision variables and the number of constraints and extend the solution search time beyond acceptable limits.

The logical constraints relate to various practical situations. In the hybrid model, three logical constraints were introduced: (28) which excludes concurrent transportation of selected products by different transport means, which eliminates the necessity of repackaging unacceptable by the customer, (29) which limits the number of participating distributors, for example, due to a limited number of available portable scanning devices for radioactivity, biological, or chemical contamination, and (30) which allows the simultaneous production, distribution, transportation, and storage of exclusively one of the set of two selected products items.

Table 2 shows indices, decision variables, and parameters used in the models. The exemplified structure of the SCM network for these models, composed of manufacturers, distributors, customers, and multimodal transport, is shown in Figure 3. The MILP and hybrid models for illustrative examples are the cost models that take into account other types of parameters, that is, time (duration of supply and service by distributor, etc.), the transport mode, distributor's capacity, capacity of transport unit, and the spatial parameters (volume/capacity occupied by the product).

The principal assumptions used in the construction of the models are as follows:

(i) Supply chain network has a structure as in Figure 3.

(ii) Supply chain management process uses information that relates to production (cost, versatility capacity, etc.), product item (volume), resources (costs, versatility, capacity, etc.), transportation (cost, time, mode, and capacity), demand (product item, time), inventory (costs, capacity, time, and versatility), and so forth.
TABLE 2: Indices, parameters, symbols, and decision variables.

\begin{tabular}{|c|c|}
\hline Symbol & Description \\
\hline \multicolumn{2}{|r|}{ Indices } \\
\hline$d$ & Product item $(d=1 \cdots D)$ \\
\hline$c$ & Customer/retailer $(c=1 \cdots C)$ \\
\hline$a$ & Factory (manufacturer) $(a=1 \cdots A)$ \\
\hline$b$ & Distributor $(b=1 \cdots B)$ \\
\hline$e$ & Mode of transportation $(e=1 \cdots E)$ \\
\hline$A$ & Number of factories \\
\hline C & Number of customers/retailers \\
\hline$B$ & Number of distributors \\
\hline$D$ & Number of product items \\
\hline E & Number of ransportation modes \\
\hline \multicolumn{2}{|r|}{ Input parameters } \\
\hline$F_{b}$ & Distributor's cost (fixed) $b$ \\
\hline$P_{d}$ & Volume/capacity occupied by product item $d$ \\
\hline$T_{d}$ & Weight of product item $d$ \\
\hline$V_{b}$ & Max capacity/volume of distributor $b$ \\
\hline$W_{a, d}$ & Production capacity of factory $a$ for product item $d$ \\
\hline$C_{a, d}$ & Cost of product item $d$ in factory $a$ \\
\hline$R_{b, d}$ & $\begin{array}{l}\text { If distributor } b \text { can supply product item } d \text { then } \\
\qquad R_{b, d}=1 \text {; otherwise } R_{b, d}=0\end{array}$ \\
\hline$T_{\mathrm{p}_{b, d}}$ & $\begin{array}{l}\text { Time required to prepare the dispatch of product } \\
\text { item } d \text { by distributor } b\end{array}$ \\
\hline$T_{c_{c, d}}$ & $\begin{array}{c}\text { Cut-off time of supply of product item } d \text { to } \\
\text { customer/retailer } c\end{array}$ \\
\hline$Z_{c, d}$ & $\begin{array}{l}\text { Size of the order for product item } d \text { placed by } \\
\text { customer } c\end{array}$ \\
\hline$Z_{\mathrm{t}_{e}}$ & $\begin{array}{l}\text { Number of transportation units using transportation } \\
\text { mode } e\end{array}$ \\
\hline$P_{\mathrm{t}_{e}}$ & $\begin{array}{l}\text { Capacity of transportation unit using transportation } \\
\text { mode } e\end{array}$ \\
\hline$T_{\mathrm{t}_{e}}$ & $\begin{array}{l}\text { Tonnage of transportation unit using transportation } \\
\text { mode } e\end{array}$ \\
\hline$T_{\mathrm{f}_{a, b, e}}$ & $\begin{array}{l}\text { Supply time from factory (manufacturer) } a \text { to } \\
\text { distributor } b \text { by transportation mode } e\end{array}$ \\
\hline$K_{1_{a, b, d, e}}$ & $\begin{array}{c}\text { Cost of supplying product item } d \text { from factory } \\
\text { (manufacturer) } a \text { to distributor } b \text { by transportation } \\
\text { mode } e \text { (variable) }\end{array}$ \\
\hline$R_{1_{a, b, e}}$ & $\begin{array}{c}\text { If factory (manufacturer) } a \text { can supply distributor } b \\
\text { using transportation mode } e \text {, then } R_{1_{a, b, e}}=1 ; \\
\text { otherwise } R_{1_{a, b, e}}=0\end{array}$ \\
\hline$A_{a, b, e}$ & $\begin{array}{l}\text { Cost of supply from factory (manufacturer) } a \text { to } \\
\text { distributor } b \text { by transportation mode } e \text { (fixed) }\end{array}$ \\
\hline $\mathrm{T}_{\mathrm{ba}_{b, c, e}}$ & $\begin{array}{l}\text { Overall cost of supply from distributor } b \text { to customer } \\
\qquad \text { by transportation mode } e\end{array}$ \\
\hline$T_{\mathrm{m}_{b, c, e}}$ & $\begin{array}{l}\text { Time of supply from distributor } b \text { to } \\
\text { customer/retailer } c \text { by transportation mode } e\end{array}$ \\
\hline$K_{2_{b, c, d, e}}$ & $\begin{array}{c}\text { Variable cost of supply of product item } d \text { from } \\
\text { distributor } b \text { to customer/retailer } c \text { by transportation } \\
\text { mode } e\end{array}$ \\
\hline$R_{2_{b, c, e}}$ & $\begin{array}{l}\text { If distributor } b \text { can supply customer/retailer } c \text { by } \\
\text { transportation mode } e \text {, then } R_{2_{b, c, e}}=1 \text {; otherwise } \\
\qquad R_{2_{b, c, e}}=0\end{array}$ \\
\hline$G_{b, c, e}$ & $\begin{array}{l}\text { Fixed cost of supply from distributor } b \text { to } \\
\text { customer/retailer } c \text { by transportation mode } e\end{array}$ \\
\hline
\end{tabular}


TABLE 2: Continued.

\begin{tabular}{|c|c|}
\hline $\mathrm{T}_{\mathrm{bg}_{b, c, e}}$ & $\begin{array}{l}\text { Overall cost of supply from distributor } b \text { to } \\
\text { customer/retailer } c \text { by transportation mode } e\end{array}$ \\
\hline $\mathrm{O}_{\mathrm{d}_{e}}$ & Environmental cost of using transportation mode $e$ \\
\hline \multicolumn{2}{|r|}{ Decision variables } \\
\hline$X_{\mathrm{p}_{a, b, d, e, c}}$ & $\begin{array}{l}\text { Quantity of a product item } d \text { supplied from factory } \\
\text { (manufacturer) } a \text { to distributor } b \text { by transportation } \\
\text { mode } e \text { to customer/retailer } c\end{array}$ \\
\hline$X_{\mathrm{o}_{a, b, d, e, c}}$ & $\begin{array}{l}\text { If supply is from factory (manufacturer) } a \text { to } \\
\text { distributor } b \text { of product } d \text { by transportation mode } e \\
\text { to customer/retailer } c \text {, then } X_{\mathrm{o}_{a, b, d, e, c}}=1 \text {, otherwise } \\
\qquad X_{\mathrm{o}_{a, b, d, e, c}}=0\end{array}$ \\
\hline$X_{c_{a, b, e}}$ & $\begin{array}{l}\text { Number of travels from factory (manufacturer) } a \text { to } \\
\text { distributor } b \text { by transportation mode } e\end{array}$ \\
\hline$Y_{\mathrm{p}_{b, c, d, e}}$ & $\begin{array}{l}\text { Quantity of product item } d \text { supplied from distributor } \\
b \text { to customer } c \text { by transportation mode } e\end{array}$ \\
\hline$Y_{\mathrm{o}_{b, c, d, e}}$ & $\begin{array}{l}\text { If supply is from distributor } b \text { to customer/retailer } c \\
\text { for product } d \text { by transportation mode } e \text {, then } \\
\qquad Y_{\mathrm{o}_{b, c, d, e}}=1 \text {; otherwise } Y_{\mathrm{o}_{b, c, d, e}}=0\end{array}$ \\
\hline$Y_{c_{b, c, e}}$ & $\begin{array}{l}\text { Number of travels from distributor } b \text { to } \\
\text { customer/retailer } c \text { by transportation mode } e\end{array}$ \\
\hline
\end{tabular}

(iii) Transportation is multimodal (a limited number of transportation units for each mode, several modes of transportation).

(iv) The environmental aspects of use of transportation units in different modes are taken into consideration.

(v) Transport batch contains various types of products.

(vi) It includes fixed and variable costs of supply (which may take the form of a linear function).

(vii) The models have integer, linear, and logical constraints (only in hybrid model).

(viii) The objective function is a linear function of cost relating to the entire supply chain.

4.1. Objective Function of the Optimization Models. The objective function (1) determines the total cost of supply chain management. It consists of five components. The first component relates to the total environmental costs (using various means of transport units). The environmental costs are dependent on the environmental levy, which can depend on fuel consumption (gasoline, oil, or gas) and carbondioxide emissions [24] and, on the other hand, on the number of courses by transportation units. The second component determines the cost of the supply from the factories to the distributors. Next element is responsible for the costs of the supply from the distributors to the customer. Another element specifies the production cost of the product item by the given factory. The last element of the objective function (1) specifies the costs (fixed) associated with the activities and processes of the distributors involved in the supply (loading, unloading, packaging, ticketing, and so on.).

The formulation of the objective function in this way allows the comprehensive optimization of the all costs in various aspects of SCM. The above objective function is the same for all models in Section 4.

$$
\begin{aligned}
& \sum_{e=1}^{E} O_{\mathrm{d}_{e}}\left(\sum_{b=1}^{B} \sum_{c=1}^{C} Y_{\mathrm{c}_{b, c, e}}+\sum_{a=1}^{A} \sum_{b=1}^{B} X_{\mathrm{c}_{a, b, e}}\right) \\
& +\sum_{b=1}^{B} \sum_{c=1}^{C} \sum_{e=1}^{E} T_{\mathrm{bg}_{b, c, e}}+\sum_{a=1}^{A} \sum_{b=1}^{B} \sum_{e=1}^{E} T_{\mathrm{ba} a, b, e} \\
& +\sum_{a=1}^{A} \sum_{d=1}^{D}\left(C_{a, d} \cdot \sum_{b=1}^{B} \sum_{c=1}^{C} \sum_{e=1}^{E} X_{\mathrm{p}_{a, b, d, e, c}}\right)+\sum_{b=1}^{B} F_{b} \cdot T_{\mathrm{c}_{b}} .
\end{aligned}
$$

4.2. Constraints. The model has the following constraints (2)-(27). Constraint (2) specifies that all supplies of product item $d$ produced by the factory $a$ to all distributors $b$ using mode of transport $e$ do not exceed the production capacity at factory $a$. Constraint (3) ensures the coverage of all customer/retailer $c$ orders $\left(Z_{c, d}\right)$ for product $d$ as a result of supply by distributors $b$. The balance of the products $d$ flow through the distributor $b$ is constraint (4). Possibility of supply by the distributor $b$ depends on its technical capabilities (5). Delivery time conditions are met by constraint (6).

The set of constraints from (7) to (11) determines the necessary means of transport for the supply.

The set of constraints (12), (13), and (14) set values of decision variables $X_{\mathrm{p}_{a, b, d, e, c}}, Y_{\mathrm{p}_{b, c, d, e}}$, based on binary decision variables $T_{c_{b}}, X_{\mathrm{o}_{a, b}}$. The remaining set of constraints (17)(27) results from the nature of the mathematical programming model.

Constraint (28) excludes transportation of selected product $d$ by different transport means $e_{1}, e_{2}, \ldots, e_{i}$.

Constraint (29) ensures that a group of products $d_{1}, d_{2}, \ldots, d_{i}$ cannot be stored in more than $B_{n}$ centers simultaneously. Constraint (30) excludes concurrent production, distribution, and transport of selected products $d_{1}$ and $d_{2}$.

The constraints above result from the practical reasons, including sales, security, marketing, contracting, or technology. Logical constraints occur especially often in a situation of strong competition.

Constraints (28)-(30) can be repeatedly reused for different pairs of product $d$ and for some of or all of factories $a$ and distributors $b$. Such a logical constraint is extremely difficult to implement in mathematical programming model. It demands that each logical constraint be replaced by many linear constraints. This sometimes leads to the explosion of the number of constraints.

Only declarative methods based on CSP make easy implementation of constraints such as (28), (29), (30) possible. The introduction to model (1)-(27) logical constraints such as (28), (29), and (30) changes its nature as well as converting it into a hybrid model (HM). This model can only be considered in the DHIF. 


$$
\begin{aligned}
& \sum_{b=1}^{B} \sum_{e=1}^{E} \sum_{c=1}^{C} R_{b, d} \cdot X_{\mathrm{p}_{a, b, d, c}} \leq W_{a, d} \quad \forall a=1 \cdots A, \forall d=1 \cdots D \\
& \sum_{b=1}^{B} \sum_{e=1}^{E}\left(Y_{\mathrm{p}_{b, c, d e}} \cdot R_{b, d}\right)=Z_{c, d} \quad \forall c=1 \cdots C, \forall d=1 \cdots D, \\
& \sum_{e=1}^{E} Y_{\mathrm{p}_{b, c, d, e}}=\sum_{a=1}^{A} \sum_{e=1}^{E} X_{\mathrm{p}_{a, b, d, e, c}} \quad \forall b=1 \cdots B, \forall d=1 \cdots D, \forall c=1 \cdots C, \\
& \sum_{d=1}^{D}\left(P_{d} \cdot \sum_{a=1}^{A} \sum_{e=1}^{E} \sum_{c=1}^{C} X_{\mathrm{p}_{a, b, d, e, c}}\right) \leq T_{c_{b}} \cdot V_{b} \quad \forall b=1 \cdots B \\
& X_{\mathrm{o}_{a, b, d, e_{1}, c}} \cdot T_{\mathrm{f}_{a, b, e}}+Y_{\mathrm{o}_{b, c, d, e_{2}}} \cdot T_{\mathrm{p}_{b, d}}+Y_{\mathrm{o}_{b, c, d, e_{2}}} \cdot T_{\mathrm{m}_{b, c, e}} \leq T_{c_{c, e}} \\
& \forall a=1 \cdots A, \forall b=1 \cdots B, \forall c=1 \cdots C, \forall d=1 \cdots D, \forall e_{1}, e_{2}=1 \cdots E, \\
& R_{1_{a, b, e}} \cdot X_{\mathrm{c}_{a, b, e}} \cdot P_{\mathrm{t}_{e}} \geq \sum_{c=1}^{C} X_{\mathrm{p}_{a, b, d, e, c}} \cdot P_{d} \quad \forall a=1 \cdots A, \forall b=1 \cdots B, \forall d=1 \cdots D, \forall e=1 \cdots E, \\
& R_{2_{b, c, e}} \cdot Y_{c_{b, c, e}} \cdot P_{\mathrm{t}_{e}} \geq Y_{\mathrm{p}_{b, c, d, e}} \cdot P_{d} \quad \forall b=1 \cdots B, \forall c=1 \cdots C, \forall d=1 \cdots D, \forall e=1 \cdots E, \\
& R a_{a, b, e} \cdot X_{\mathrm{c}_{a, b, e}} \cdot T_{\mathrm{t}_{e}} \geq \sum_{c=1}^{C} X_{\mathrm{p}_{a, b, d, e, c}} \cdot T_{d} \quad \forall a=1 \cdots A, \forall b=1 \cdots B, \forall d=1 \cdots D, \forall e=1 \cdots E, \\
& R b_{b, c, e} \cdot Y_{c_{b, c, e}} \cdot T_{\mathrm{t}_{e}} \geq Y_{\mathrm{p}_{b, c, d, e}} \cdot T_{d} \quad \forall b=1 \cdots B, \forall c=1 \cdots C, \forall d=1 \cdots D, \quad \forall e=1 \cdots E, \\
& \sum_{a=1}^{A} \sum_{b=1}^{B} X_{c_{a, b, e}}+\sum_{c=1}^{C} \sum_{b=1}^{B} Y_{c, b, e} \leq Z_{\mathrm{t}_{e}} \quad \forall e=1 \cdots E \\
& \sum_{a=1}^{A} \sum_{e=1}^{E} X_{\mathrm{c}_{a, b, e}} \leq C W \cdot T_{\mathrm{c}_{b}} \quad \forall b=1 \cdots B \\
& X_{\mathrm{p}_{a, b, d, e, c}} \leq C W \cdot X_{\mathrm{o}_{a, b, d, e, c}} \quad \forall a=1 \cdots A, \forall b=1 \cdots B, \forall d=1 \cdots D, \forall e=1 \cdots E, \forall c=1 \cdots C, \\
& Y_{\mathrm{p}_{b, c, d, e}} \leq C W \cdot Y_{\mathrm{o}_{b, c, d, e}} \quad \forall b=1 \cdots b, \forall c=1 \cdots C, \forall d=1 \cdots D, \forall e=1 \cdots E, \\
& T_{\mathrm{ba} a, b, e}=A_{a, b, e} \cdot X_{\mathrm{c}_{a, b, e}}+\sum_{d=1}^{D} K_{1_{a, b, d, e}} \cdot \sum_{c=1}^{C} X_{\mathrm{p}_{a, b, d, e, c}} \quad \forall a=1 \cdots A, \forall b=1 \cdots B, \forall e=1 \cdots E, \\
& T_{\mathrm{bg}_{b, c, e}}=\sum_{d=1}^{D} K_{2_{b, c, d, e}} \cdot Y_{\mathrm{p}_{b, c, d e}}+G_{b, c, e} \cdot Y_{c_{b, c, e}} \quad \forall b=1 \cdots B, \forall c=1 \cdots C, \forall e=1 \cdots E, \\
& X_{\mathrm{p}_{a, b, c, e, d}} \geq 0 \quad \forall a=1 \cdots A, \forall b=1 \cdots B, \forall c=1 \cdots C, \forall e=1 \cdots E, \forall d=1 \cdots D, \\
& X_{\mathrm{p}_{a, b, c, e, d}} \in C \quad \forall a=1 \cdots A, \forall b=1 \cdots B, \forall c=1 \cdots C, \forall e=1 \cdots E, \forall d=1 \cdots D, \\
& X_{\mathrm{o}_{a, b, c, e, d}} \in\{0,1\} \quad \forall a=1 \cdots A, \forall b=1 \cdots B, \forall c=1 \cdots C, \forall e=1 \cdots E, \forall d=1 \cdots D \\
& X_{\mathrm{c}_{a, b, e}} \geq 0 \quad \forall a=1 \cdots A, \forall b=1 \cdots B, \forall e=1 \cdots E \\
& X_{c_{a, b, e}} \in C \quad \forall a=1 \cdots A, \forall b=1 \cdots B, \forall e=1 \cdots E \text {, } \\
& Y_{\mathrm{p}_{b, c, d, e}} \geq 0 \quad \forall b=1 \cdots B, \forall c=1 \cdots C, \forall d=1 \cdots D, \forall e=1 \cdots E, \\
& Y_{\mathrm{p}_{b, c, d, e}} \in C \quad \forall b=1 \cdots b, \forall c=1 \cdots C, \forall d=1 \cdots D, \forall e=1 \cdots E, \\
& Y_{\mathrm{o}_{b, c, d, e}} \in\{0,1\} \quad \forall b=1 \cdots B, \forall c=1 \cdots C, \forall d=1 \cdots D, \forall e=1 \cdots E
\end{aligned}
$$




$$
\begin{gathered}
Y_{c_{b, c, e}} \geq 0 \quad \forall b=1 \cdots B, \forall c=1 \cdots C, \forall e=1 \cdots E, \\
Y_{c_{b, c, e}} \in C \quad \forall b=1 \cdots B, \forall c=1 \cdots C, \forall e=1 \cdots E, \\
T_{c_{b}} \in\{0,1\} \quad \forall b=1 \cdots B, \\
\operatorname{LogicS}\left(d, b_{1}, b_{2}\right) \quad \forall d \in 1 \cdots D, \forall b_{1}, b_{2} \in 1 \cdots B, b_{1} \neq b_{2}, \\
\operatorname{LogicS}_{n}\left(d_{1}, \ldots, d_{i}, b, B_{n}\right) \quad \forall d_{1}, d_{2}, \ldots, d_{i} \in 1 \cdots D, \forall b \in 1 \cdots B, d_{i} \neq d_{j}, \\
\operatorname{LogicExl}\left(d_{1}, d_{2}, b, a, e\right) \quad \forall d_{1}, d_{2} \in 1 \cdots D, \forall b \in 1 \cdots B, \forall a \in 1 \cdots A, \forall e \in 1 \cdots E, d_{1} \neq d_{2} .
\end{gathered}
$$

4.3. The Transformation of Illustrative Models. The possibility of transformation of the problem is an important feature of the proposed method. Multidimensional transformation $\mathrm{T}^{\mathrm{CLP}}$ (Section 3.1) was used in the illustrative examples as a presolving method. The representation of the problem was altered using S-structure of the problem and D-data. Our goal was the transformation of the problem to make a significant reduction in its size without changing the problem. It consisted in changing the representation of the problem. Any acceptable routes connecting factories, distributors, and customers were generated based on the set of facts relating to orders and supply chain structure.

Then, the specific values of parameters like manufacturers $a$, distributors $b$, products $d$, mode of transport form factory to distributor $e_{1}$, mode of transport form distributor to customer $e_{2}$, and customers $c$ (Table 2) were assigned to each of the acceptable routes. Thus, only one parameter, $X_{\mathrm{p}}$ (the volume of supplies), needs to be determined.

This means that six sets of parameters more were to be determined before the transformation. In this step the sets of decision variables $X^{\mathrm{T}}$ and constraints $\mathrm{C}^{\mathrm{T}}$ were subject to change. Thereby, the obtained model after the transformation MILP_T (1T)-(17T) has different decision variables and different constraints than those in the MILP (1)-(27). Some of the decision variables are redundant; other variables are subject to aggregation. This results in a dramatic reduction in their number. Decision variables before and after the transformation are shown in Table 3(a). The transformation also reduces or eliminates some of the constraints of the model. Thus, constraints (4), (6), (12), (13), and (14), present in the MILP (1)-(27) and shown in Section 4.2, are redundant in the MILP_T. Balance constraint (4) is unnecessary because the route defines the specific distribution center. Only those routes that meet the time constraints are generated; therefore constraint (6) does not make sense. Binarity ensures whether or not the route occurs; thus constraint (12) is redundant. Reduction of certain variables also affects the reduction of constraints, hence the lack of constraints (13) and (14), in the model. Constraints before and after the transformation are shown in Table 3(b).

In the following step of the transformation, the properties of the CLP including constraint propagation were used to find, based on the domains (Tables 4 and 5), certain quantities and create a new set of additional constraints $\mathrm{C}^{\mathrm{AT}}$ (Table 6). Therefore, transformed model (MILP_T) was extended to additional constraints (1AC)-(6AC). These constraints affect the efficiency of the search for a solution by narrowing down the search area. It is obvious that the transformed model (MILP_T) can be extended from logical constraints (28)-(30) to a hybrid model (HM).

The proposed transformation improves the efficiency and effectiveness of the constraint propagation by reduction of the number of backtracks. It will be explicitly shown in the numerical experiments in Section 4.6.

$$
\begin{aligned}
& \sum_{e=1}^{E} O_{\mathrm{d} e} \cdot\left(\sum_{a=1}^{A} \sum_{b=1}^{B} X_{\mathrm{c}_{a, b, e}}+\sum_{b=1}^{b} \sum_{c=1}^{c} Y_{\mathrm{c}_{b, c, e}}\right)+\sum_{a=1}^{A} \sum_{d=1}^{D} \sum_{b=1}^{B} \sum_{c=1}^{C} \sum_{e_{1}=1}^{E} \sum_{e_{2}=1}^{E}\left(X_{\mathrm{p}_{a, d, b, c, e_{1}, e_{2}}}^{\mathrm{T}} \cdot K_{\mathrm{z}_{a, d, b, c, e}, e_{2}}\right)+\sum_{a=1}^{A} \sum_{b=1}^{B} \sum_{e=1}^{O}\left(X_{\mathrm{c}_{a, b, e}} \cdot K_{\mathrm{sc}_{a, b, e}}\right) \\
& \quad+\sum_{b=1}^{B} \sum_{c=1}^{C} \sum_{e=1}^{E}\left(Y_{c_{b, c, e}} \cdot K_{\mathrm{sm}_{b, c, e}}\right)+\sum_{b=1}^{B} T_{c_{b}} \cdot F_{b} \\
& \sum_{b=1}^{b} \sum_{c=1}^{C} \sum_{e_{1}=1}^{E} \sum_{e_{2}=1}^{E} X_{\mathrm{p}_{a, d, b, c, e_{1}, e_{2}}}^{\mathrm{T}} \leq W_{a, d} \quad \forall a=1 \cdots A, \forall d=1 \cdots D \\
& \sum_{a=1}^{A} \sum_{b=1}^{B} \sum_{e_{1}=1}^{E} \sum_{e_{2}=1}^{E} X_{\mathrm{p}_{a, d, b, e_{1}, e_{2}}}^{\mathrm{T}}=Z_{c, d} \quad \forall c=1 \cdots C, \forall d=1 \cdots D, \\
& \sum_{a=1}^{A} \sum_{d=1}^{D} \sum_{c=1}^{C} \sum_{e_{1}=1}^{E} \sum_{e_{2}=1}^{E} X_{\mathrm{p}_{a, d, b, e_{1}, e_{2}}^{\mathrm{T}}}^{\mathrm{T}} \leq V_{b} \cdot T_{c_{b}} \quad \forall b=1 \cdots B,
\end{aligned}
$$




$$
\begin{aligned}
& \sum_{d=1}^{D} \sum_{c=1}^{C} \sum_{e_{2}=1}^{E}\left(P_{d} \cdot X_{\mathrm{p}_{a, d, b, c, e_{1}, e_{2}}^{\mathrm{T}}}^{\mathrm{T}}\right) \leq P_{\mathrm{t}_{e_{1}}} \cdot X_{\mathrm{c}_{a, b, e_{1}}} \quad \forall a=1 \cdots A, \forall b=1 \cdots B, \forall e_{1}=1 \cdots E, \\
& \sum_{a=1}^{A} \sum_{d=1}^{D} \sum_{e_{1}=1}^{E}\left(P_{d} \cdot X_{\mathrm{p}_{a, d, b, c, e_{1}, e_{2}}}^{\mathrm{T}}\right) \leq P_{\mathrm{t}_{e_{2}}} \cdot Y_{\mathrm{c}_{b, c, e_{2}}} \quad \forall b=1 \cdots B, \forall c=1 \cdots C, \forall e_{2}=1 \cdots E, \\
& \sum_{d=1}^{D} \sum_{c=1}^{C} \sum_{e_{2}=1}^{E}\left(T_{d} \cdot X_{\mathrm{p}_{a, d, b, c, e_{1}, e_{2}}}^{\mathrm{T}}\right) \leq T_{\mathrm{t}_{e_{1}}} \cdot X_{\mathrm{c}_{a, b, e_{1}}} \quad \forall a=1 \cdots A, \forall b=1 \cdots B, \forall e_{1}=1 \cdots E, \\
& \sum_{a=1}^{A} \sum_{d=1}^{D} \sum_{e_{2}=1}^{E}\left(T_{d} \cdot X_{\mathrm{p}_{a, d, b, c, e_{1}, e_{2}}}^{\mathrm{T}}\right) \leq T_{\mathrm{t}_{e_{1}}} \cdot Y_{\mathrm{c}_{b, c, e_{1}}} \quad \forall b=1 \cdots B, \forall c=1 \cdots C, \forall e_{1}=1 \cdots E, \\
& \sum_{a=1}^{A} \sum_{b=1}^{b} X_{\mathrm{c}_{i, s, d}}+\sum_{b=1}^{B} \sum_{c=1}^{C} Y_{\mathrm{c}_{b, c, e}} \leq Z_{\mathrm{t}_{e}} \quad \forall e=1 \cdots E \\
& X_{\mathrm{p}_{a, d, b, c, e_{1}, e_{2}}^{\mathrm{T}}}^{\mathrm{T}}=0 \quad \forall X_{\mathrm{d}_{a, d, b, c, e_{1}, e_{2}}}=0, \forall a=1 \cdots A, \forall d=1 \cdots D, \forall b=1 \cdots B, \forall c=1 \cdots C, \forall e_{1}, e_{2}=1 \cdots E \\
& X_{a, d, b, c, e_{1}, e_{2}}^{\mathrm{T}} \geq 0 \quad \forall a=1 \cdots A, \forall d=1 \cdots D, \forall b=1 \cdots B, \forall c=1 \cdots C, \forall e_{1}, e_{2}=1 \cdots E, \\
& X_{\mathrm{c}_{a, b, e}} \geq 0 \quad \forall a=1 \cdots A, \forall b=1 \cdots B, \forall e=1 \cdots E, \\
& Y_{c_{b, c, e}} \geq 0 \quad \forall b=1 \cdots B, \forall c=1 \cdots C, \forall e=1 \cdots E \text {, } \\
& X_{\mathrm{p}_{a, d, b, c, e_{1}, e_{2}}^{\mathrm{T}}}^{\mathrm{T}} \in C \quad \forall a=1 \cdots A, \forall d=1 \cdots D, \forall b=1 \cdots B, \forall c=1 \cdots C, \forall e_{1}, e_{2}=1 \cdots E, \\
& X_{c_{a, b, e}} \in C \quad \forall a=1 \cdots A, \forall b=1 \cdots B, \forall e=1 \cdots E \\
& Y_{c_{b, c, e}} \in C \quad \forall b=1 \cdots B, \forall c=1 \cdots C, \forall e=1 \cdots E, \\
& T_{c_{b}} \in\{0,1\} \quad \forall b=1 \cdots B \\
& \operatorname{LogicS}^{\mathrm{T}}\left(d, b_{1}, b_{2}\right) \quad \forall d \in 1 \cdots D, \forall b_{1}, b_{2} \in 1 \cdots B, s_{1} \neq s_{2}, \\
& \operatorname{LogicS}_{n}^{\mathrm{T}}\left(d_{1}, \ldots, d_{i}, b, B_{n}\right) \quad \forall d_{1}, d_{2}, \ldots, k_{i} \in 1 \cdots O, \forall b \in 1 \cdots B, d_{i} \neq d_{j}, \\
& \operatorname{LogicExl}^{\mathrm{T}}\left(d_{1}, d_{2}, b, a, e\right) \quad \forall d_{1}, d_{2} \in 1 \cdots D, \forall b \in 1 \cdots B, \forall e \in 1 \cdots E, \forall a \in 1 \cdots A, d_{1} \neq d_{2}, \\
& \sum_{a=1}^{A} \sum_{b=1}^{B} X_{c_{a, b, e}}+\sum_{b=1}^{B} \sum_{c=1}^{C} Y_{c_{b, c e}} \geq R_{\min _{e}} \quad \forall e=1 \cdots E, \\
& \sum_{a=1}^{A} \sum_{b=1}^{B} X_{\mathrm{c}_{a, b, e}}+\sum_{b=1}^{B} \sum_{c=1}^{C} Y_{\mathrm{c}_{b, c, e}} \leq R_{\max _{e}} \quad \forall e=1 \cdots E, \\
& \sum_{a=1}^{A} \sum_{b=1}^{B} \sum_{e=1}^{E} X_{\mathrm{c}_{a, b, e}} \geq \mathrm{F}_{-} \mathrm{C} \text {, } \\
& \sum_{b=1}^{B} \sum_{c=1}^{C} \sum_{E=1}^{E} Y_{c_{b, c, e}} \geq C_{-} \text {C, } \\
& \sum_{a=1}^{A} \sum_{b=1}^{B} \sum_{e=1}^{E} X_{c_{a, b, e}}+\sum_{b=1}^{b} \sum_{c=1}^{C} \sum_{e=1}^{E} Y_{c_{b, c, e}} \geq \min _{-} \mathrm{TU}, \\
& \sum_{b=1}^{B} T_{c_{b}} \geq \mathrm{Cn}
\end{aligned}
$$


4.4. Soft Constraints. Constraints of the transformed model can be satisfied in the hard or soft form. This is due to practical circumstances. For some of the constraints, it is difficult to satisfy in full (hard), due to limited resources, time, technology, and so forth or their fulfillment requires unacceptable computational effort.

In the highly competitive environment we need to know how much "costs" exceed a given constraint or fulfill it in part.

Such constraints can be satisfied in an approximate way, that is, in the soft form. In this case, how this affects the value of the objective function should be determined.

The softening of constraints in this approach is a process which contains the following: (a) changing their form and (b) introducing a modification to the objective function.
For instance, four constraints of the transformed model of illustrative example (9T), (18T), (19T), and (20T) have been converted into soft form, (2S), (3S), (4S), and (5S).

Constraint (2S) allows exceeding the limit of available number of transportation units using transportation mode $e$.

Constraints (3S), (4S), and (5S) allow the possibility of not fulfilling exclusions imposed by logical constraints (18T), (19T), and (20T).

For this purpose, the three additional components to the objective function have been introduced as a penalty for violation of constraint (1S). Table 7 shows parameters and decision variables necessary to modify the model with soft constraints.

$$
\begin{gathered}
\sum_{a=1}^{A} K_{\mathrm{ri}_{a}} \cdot X_{\mathrm{ri}_{a}}+\sum_{e=1}^{E} K_{\mathrm{rd}_{e}} \cdot X_{\mathrm{rd}_{e}}+\sum_{b=1}^{B} K_{\mathrm{rs}_{b}} \cdot X_{\mathrm{rs}_{b}}, \\
\sum_{a=1}^{A} \sum_{b=1}^{B} X_{\mathrm{c}_{a, b, e}}+\sum_{b=1}^{B} \sum_{c=1}^{C} Y_{c_{b, c},} \leq Z_{\mathrm{t}_{e}}+X_{\mathrm{rd}_{e}} \quad \forall e=1 \cdots E, \\
\operatorname{SoftLogicS}^{\mathrm{T}}\left(d, b_{1}, b_{2}\right) \quad \forall d \in 1 \cdots D, \forall b_{1}, b_{2} \in 1 \cdots B, b_{1} \neq b_{2}, \\
\operatorname{SoftLogicS}_{n}^{\mathrm{T}}\left(d_{1}, \ldots, d_{i}, b, B_{n}\right) \quad \forall d_{1}, d_{2}, \ldots, d_{i} \in 1 \cdots D, \forall b \in 1 \cdots B, d_{i} \neq d_{j}, \\
\text { SoftLogicExl }{ }^{\mathrm{T}}\left(d_{1}, d_{2}, b, a, e\right) \quad \forall d_{1}, d_{2} \in 1 \cdots D, \forall b \in 1 \cdots B, \forall e \in 1 \cdots E, \forall a \in 1 \cdots A, d_{1} \neq d_{2} .
\end{gathered}
$$

4.5. Decision Support. The implementation of the presented models by DHIF allows the decision support in the following areas of the supply chain management (including but not limited to):

(i) The cost optimization of the supply chain management (Table 8).

(ii) The volume/capacity of distributor depots/storehouses and the investigation of their influence on the overall costs (Figure 4(a), Table 9).

(iii) The selection of the capacity of transportation units and modes, the transport fleet number for specific entire costs (Table 10, Figure 4(b)).

(iv) The selection of a set of routes for cost optimization (Figure 5(a)).

(v) Implementation of logical constraints in deliveries that result from technology, security, marketing, competition, and sales reasons (Table $8, \mathrm{Q}_{1}, \mathrm{Q}_{2}, \mathrm{Q}_{3}$, and Figure 5(b)).

(vi) Costs exceeding the available means of transport (soft constraints), Table 11.

Supporting this type of decision is extremely important in a highly competitive environment. Making optimal choices under existing constraints within an acceptable time gives you a competitive advantage.
4.6. Computational Experiments and Analysis. A number of numerical experiments were conducted to verify and evaluate the proposed method, models, and framework. All the numerical examples refer to the supply chain with twenty customers $(c=1 \cdots 20)$, four distributors $(b=1 \cdots 4)$, five manufacturers $(a=1 \cdots 5)$, and four modes of transport $(e=1 \cdots 4)$. In the first phase of the experiments, the optimization was performed for ten examples, $\mathrm{Pn}_{1}-\mathrm{Pn}_{10}$, for the MILP model (1)-(27). The computational examples vary in the number of orders $(N)$. The capacity available to the distributors $b\left(V_{1}=V_{2}=V_{3}=V_{4}=2000\right)$ and the number of transport units $\left(Z_{\mathrm{t}_{1}}=40, Z_{\mathrm{t}_{2}}=\right.$ $\left.60, Z_{\mathrm{t}_{1}}=60, Z_{\mathrm{t}_{1}}=70\right)$ using the mode of transport $e$ were the same for all the examples. The experiments were conducted to show the effectiveness and efficiency of the declarative hybrid method and implementation framework and their advantage over other methods. In order to prove this, model (1)-(27) was implemented in both the declarative hybrid implementation framework (DHIF) (MILP_T) and the mathematical programming environment (MILP). The experiments that follow were conducted to optimize examples $\mathrm{Pn}_{11}-\mathrm{Pn}_{20}$, which are the implementations of the hybrid model (HM) (1)-(30) in the DHIF. Examples $\mathrm{Pn}_{11}-$ $\mathrm{Pn}_{20}$ were obtained from $\mathrm{Pn}_{1}-\mathrm{Pn}_{10}$ by the addition of logical constraints: (28) for $\mathrm{Q}_{3}$ series, (29) for $\mathrm{Q}_{2}$ series, and (30) for $\mathrm{Q}_{1}$ series. For example, $\mathrm{Pn}_{1}-\mathrm{Pn}_{3}$ the optimal solution for the MILP_T implementation in the DHIF was found faster than that for the MILP implementation in mathematical 
TABLE 3: (a) Decision variables used in the MILP and MILP_T models. (b) Constraints used in the MILP and MILP_T models.

(a)

\begin{tabular}{|c|c|c|}
\hline $\begin{array}{l}\text { MILP } \\
\left(X^{\mathrm{O}}\right)\end{array}$ & $\begin{array}{l}\text { MILP_T } \\
\left(X^{\mathrm{T}}\right)\end{array}$ & Description of the decision variables after the multidimensional transformation \\
\hline $\begin{array}{l}X_{\mathrm{p}_{a, b, c, e_{1}, d}} \\
Y_{\mathrm{p}_{b, c, d, e_{2}}}\end{array}$ & $X_{\mathrm{p}_{a, d, b, c, e_{1}, e_{2}}^{\mathrm{T}}}^{\mathrm{T}}$ & $\begin{array}{l}\text { Decision variable } X_{\mathrm{p}}^{\mathrm{T}} \text {, unlike the initial decision variables } X_{\mathrm{p}}, Y_{\mathrm{p}} \text {, is generated only for technologically } \\
\text { possible indices combinations; it defines the allocation size of product } d \text { to the route of deliveries }\end{array}$ \\
\hline$X_{\mathrm{o}_{a, b, d, e, c}}$ & Unnecessary & After transformation replaced by the appropriate factor for the route, generated by the CLP \\
\hline$X_{c_{a, b, e}}$ & $X_{c_{a, b, e}}$ & Without change, the same sense \\
\hline$Y_{\mathrm{a}_{s, j, k, d}}$ & Unnecessary & After transformation replaced by the appropriate factor for the route, generated by the CLP \\
\hline$Y_{\mathrm{o}_{b, c, e}}$ & $Y_{\mathrm{o}_{b, c, e}}$ & Without change, the same sense \\
\hline$T_{c_{b}}$ & $T_{c_{b}}$ & Without change, the same sense \\
\hline
\end{tabular}

(b)

\begin{tabular}{|c|c|c|}
\hline MILP & MILP_T & $\begin{array}{l}\text { Description of the constraints after the multidimensional } \\
\text { transformation }\end{array}$ \\
\hline$\left(\mathrm{C}^{\mathrm{O}}\right)$ & $\left(\mathrm{C}^{\mathrm{T}}\right)$ & \\
\hline (1) & $(1 \mathrm{~T})$ & Objective function (the same meaning) \\
\hline$(2)$ & $(2 \mathrm{~T})$ & The manufacturer's production capacity (the same meaning) \\
\hline (3) & $(3 \mathrm{~T})$ & The customer's demands (the same meaning) \\
\hline (4) & Unnecessary & $\begin{array}{l}\text { Redundant after transformation (results from the decision variable } \\
\qquad X^{\mathrm{T}} \text { ) }\end{array}$ \\
\hline (5) & $(4 \mathrm{~T})$ & The distributor's capacity/volume (the same meaning) \\
\hline (6) & Unnecessary & $\begin{array}{c}\text { Redundant after transformation } \\
\text { During the transformation generates routes that meet the time } \\
\text { constraint }\end{array}$ \\
\hline (7), (8), (9), (10) & $(5 \mathrm{~T}),(6 \mathrm{~T}),(7 \mathrm{~T}),(8 \mathrm{~T})$ & The appropriate number of means of transport (the same sense) \\
\hline (11) & $(9 \mathrm{~T})$ & $\begin{array}{l}\text { The sum of the number of means of transport used does not exceed } \\
\text { the limit of their number (the same meaning) }\end{array}$ \\
\hline (12) & Unnecessary & Redundant after transformation (ensures constraint (4)) \\
\hline (13), (14) & Unnecessary & $\begin{array}{l}\text { Redundant after transformation } \\
\text { After transformation replaced by the appropriate factor for the route }\end{array}$ \\
\hline (15), (16) & Unnecessary & $\begin{array}{l}\text { Redundant after transformation } \\
\text { Calculate the auxiliary parameters, performed by CLP in } \\
\text { transformation }\end{array}$ \\
\hline$(17)-(27)$ & (11)-(17) & Integrity and binarity \\
\hline$(28)-(30)$ & $(18 \mathrm{~T})-(20 \mathrm{~T})$ & Logic constraints (the same meaning) \\
\hline- & $(10)$ & Resetting the nonexisting routes after transformation \\
\hline & $\left(\mathrm{C}^{\mathrm{AT}}\right)$ & \\
\hline- & $(1 \mathrm{AC})-(6 \mathrm{AC})$ & Additional constraints increase the range of propagation \\
\hline
\end{tabular}

programming environment (Table 7). For illustrative models, the use of this method allows obtaining optimal solutions eight to one hundred times faster, with a significant reduction in the size of the combinatorial problem. For example, $\mathrm{Pn}_{4}-$ $\mathrm{Pn}_{10}$ the optimal solution was found only for the MILP_T implementation within an acceptable time. Owing to the DHIF platform with the multidimensional transformation, the number of decision variables for the above examples was reduced even hundredfold and the number of constrains decreased at least 30 -fold (Table 8). It is clear that optimization of the hybrid model (HM) was, due to its logical constraints, only possible using the declarative hybrid implementation framework. Also, the presented declarative hybrid method brought the expected results for this model. In further experiments, parameters such as number of transport units, distributor capacity were changed to observe the effect of this change on the optimal value of the objective function. Thus, Figure 4(a) shows the effect of capacity $V$ (distributor) on the objective function $\mathrm{Fc}^{\mathrm{opt}}$. Actual use of the distributor capacity $\left(V_{x_{1}}, V_{x_{2}}, V_{x_{3}}\right)$ for particular distributors is shown in Table 9. In contrast, Table 10 and Figure 4(b) show the effect of the parameter $Z_{t_{e}}$ on the objective function $\mathrm{Fc}^{\mathrm{opt}}$.

Table 11 shows the results of the optimization model MILP_T with the constraint on the number of means of transport in hard version (9T) and soft version (2S). Computation times for the model with a soft constraint are slightly 
TABLE 4: Input parameters determined by CLP for MILP_T.

\begin{tabular}{|c|c|}
\hline Symbol & Description \\
\hline$K_{z_{a, d, b, c, e_{1}, e_{2}}}$ & $\begin{array}{l}\text { The variable cost of supply of product item } d \text { from } \\
\text { factory } a \text { to customer } c \text { via distributor } b \text { using } \\
\text { modes of transportation } e_{1} \text { and } e_{2} \text { (CLP, based on } \\
\text { fixed data) }\end{array}$ \\
\hline$X_{\mathrm{d}_{a, d, b, c, e}, e_{2}}$ & $\begin{array}{l}\text { The binary parameter which is equal to } 1 \text { if there is } \\
\text { a route for specific } a, d, b, c, e_{1}, e_{2} \text { and zero } \\
\text { otherwise }\end{array}$ \\
\hline$K_{\mathrm{sc}_{a a, b, e}}$ & $\begin{array}{l}\text { The cost of the course/route from the factory } a \text { to } \\
\text { distributor } b \text { using mode of transportation } e \text { (CLP, } \\
\text { based on fixed data) }\end{array}$ \\
\hline$K_{\mathrm{sm}_{b, c, e}}$ & $\begin{array}{l}\text { The cost of the course/route from the distributor } b \\
\text { to customer } c \text { using mode of transportation } e \\
\text { (CLP, based on fixed data) }\end{array}$ \\
\hline
\end{tabular}

TABle 5: Figures determined by CLP for MILP_T.

\begin{tabular}{ll}
\hline Symbol & Description \\
\hline$R_{\text {min }_{e}}$ & $\begin{array}{l}\text { Minimum number of transport units } e \\
\text { (CLP-constraint propagation) }\end{array}$ \\
$R_{\text {max }_{e}}$ & $\begin{array}{l}\text { Maximum number of transport units } e \\
\text { (CLP-constraint propagation) }\end{array}$ \\
F_C & $\begin{array}{l}\text { Minimum number of transportation units in the } \\
\text { route Factories - Distributors (CLP-constraint } \\
\text { propagation) }\end{array}$ \\
& $\begin{array}{l}\text { Minimum number of transportation units in the } \\
\text { route, distributors - customers (CLP-constraint } \\
\text { propagation) }\end{array}$ \\
& $\begin{array}{l}\text { Minimum number of transportation units } \\
\text { (CLP-constraint propagation) }\end{array}$ \\
Min_TU & $\begin{array}{l}\text { Minimum number of active distributors } \\
\text { (CLP-constraint propagation) }\end{array}$ \\
Cn &
\end{tabular}

TABLE 6: Additional constraints for MILP_T.

\begin{tabular}{|c|c|}
\hline Constraints & Description \\
\hline$(1 \mathrm{AC})$ & $\begin{array}{l}\text { Narrowing the size of the transport unit domain } \\
\text { from the bottom }\end{array}$ \\
\hline$(2 \mathrm{AC})$ & $\begin{array}{l}\text { Narrowing the size of the transport unit domain } \\
\text { from the top }\end{array}$ \\
\hline$(3 \mathrm{AC})$ & $\begin{array}{l}\text { The minimum number of all transport unit types } \\
\text { necessary for the shipment from the factory to the } \\
\text { distributors/distribution centers }\end{array}$ \\
\hline$(4 \mathrm{AC})$ & $\begin{array}{l}\text { The minimum number of all transport unit types } \\
\text { necessary for the shipment from the } \\
\text { distributors/distribution centers to customers }\end{array}$ \\
\hline$(5 \mathrm{AC})$ & $\begin{array}{l}\text { The minimum number of transportation units in } \\
\text { routes }\end{array}$ \\
\hline$(6 \mathrm{AC})$ & $\begin{array}{l}\text { The number of working distributors/distribution } \\
\text { centers }\end{array}$ \\
\hline
\end{tabular}

worse than the version with limited hard constraint. The differences are fully acceptable. You can also see the impact of the application of constraint (2S) on the objective function. And, most importantly, we managed to find the optimum solution, for example, $\mathrm{P}_{3}(30)$ in the model MILP_T with constraint (2S).
TABLE 7

\begin{tabular}{cc}
\hline & Input parameters \\
\hline$K_{\mathrm{rs}_{b}}$ & $\begin{array}{r}\text { Penalty coefficient if two selected type of products are } \\
\text { stored together, despite the ban }\end{array}$ \\
$K_{\mathrm{rd}_{e}}$ & $\begin{array}{c}\text { Penalty coefficient for the use of means of transport } \\
\text { over the limit }\end{array}$ \\
$K_{\mathrm{ri}_{a}}$ & $\begin{array}{c}\text { Penalty coefficient if two selected types of products are } \\
\text { produced together, despite the ban }\end{array}$ \\
\hline & Decision variables \\
\hline$X_{\mathrm{rs}_{b}}$ & $\begin{array}{c}\text { How many pairs of type of products are stored together } \\
\text { in the distribution center } b \text { despite the ban? }\end{array}$ \\
$X_{\mathrm{rd}_{e}}$ & $\begin{array}{c}\text { How many modes of transport } e \text { are used over the limit? } \\
X_{\mathrm{ri}_{a}}\end{array}$ \\
\hline
\end{tabular}

TABLE 8: The results of numerical examples for both methods.

(a)

\begin{tabular}{lcccc}
\hline & \multicolumn{4}{c}{ DHIP } \\
$\operatorname{Pn}(N)$ & $F^{\text {opt }}$ & $T$ & $V\left(V_{\text {int }}\right)$ & $C$ \\
\hline $\mathrm{Pn}_{1}(10)$ & 165180 & 10 & $818(788)$ & 1367 \\
$\mathrm{Pn}_{2}(20)$ & 570715 & 35 & $987(948)$ & 1376 \\
$\mathrm{Pn}_{3}(30)$ & 897015 & 71 & $1264(1220)$ & 1381 \\
$\mathrm{Pn}_{4}(40)$ & 1564990 & 145 & $1536(1492)$ & 1381 \\
$\mathrm{Pn}_{5}(50)$ & 2619310 & 201 & $1696(1652)$ & 1381 \\
$\mathrm{Pn}_{6}(60)$ & 2963030 & 254 & $2080(2036)$ & 1381 \\
$\mathrm{Pn}_{7}(70)$ & 3860300 & 290 & $2240(2196)$ & 1381 \\
$\mathrm{Pn}_{8}(80)$ & 4786960 & 320 & $2512(2468)$ & 1381 \\
$\mathrm{Pn}_{9}(90)$ & 5964200 & 321 & $2779(2736)$ & 1380 \\
$\mathrm{Pn}_{10}(100)$ & 7397970 & 367 & $2939(2896)$ & 1380 \\
\hline
\end{tabular}

(b)

\begin{tabular}{lcccc}
\hline & \multicolumn{4}{c}{ Mathematical programming } \\
$\mathrm{Pn}(N)$ & $\mathrm{Fc}^{\text {opt }}$ & $T$ & $V\left(V_{\text {int }}\right)$ & $C$ \\
\hline $\mathrm{Pn}_{1}(10)$ & 165180 & 84 & $80117(79204)$ & 47426 \\
$\mathrm{Pn}_{2}(20)$ & 570715 & 752 & $80117(79204)$ & 50626 \\
$\mathrm{Pn}_{3}(30)$ & 897015 & 7248 & $80117(79204)$ & 53826 \\
$\mathrm{Pn}_{4}(40)$ & $1613453^{*}$ & $600^{* *}$ & $80117(79204)$ & 57026 \\
$\mathrm{Pn}_{5}(50)$ & $2699720^{*}$ & $600^{* *}$ & $80117(79204)$ & 60226 \\
$\mathrm{Pn}_{6}(60)$ & $3003310^{*}$ & $600^{* *}$ & $80117(79204)$ & 63426 \\
$\mathrm{Pn}_{7}(70)$ & $4062950^{*}$ & $600^{* *}$ & $80117(79204)$ & 66626 \\
$\mathrm{Pn}_{8}(80)$ & $5092400^{*}$ & $600^{* *}$ & $80117(79204)$ & 69826 \\
$\mathrm{Pn}_{9}(90)$ & $6543450^{*}$ & $600^{* *}$ & $80117(79204)$ & 73026 \\
$\mathrm{Pn}_{10}(100)$ & $8213450^{*}$ & $600^{* *}$ & $80117(79204)$ & 76226 \\
\hline
\end{tabular}

(c)

\begin{tabular}{lcccc}
\hline & \multicolumn{4}{c}{$\mathrm{Q}_{1}$} \\
$\mathrm{PH}(N)$ & & $\mathrm{H}$ HM & \\
& $\mathrm{Fc}^{\mathrm{opt}}$ & $T$ & $V\left(V_{\text {int }}\right)$ & $C$ \\
\hline $\mathrm{Pn}_{11}(10)$ & 193590 & 17 & $829(800)$ & 1384 \\
$\mathrm{Pn}_{12}(20)$ & 641315 & 45 & $999(960)$ & 1394 \\
$\operatorname{Pn}_{13}(30)$ & 1000770 & 82 & $1276(1232)$ & 1399 \\
$\operatorname{Pn}_{14}(40)$ & 1677290 & 198 & $1548(1504)$ & 1399 \\
\hline
\end{tabular}


(c) Continued.

\begin{tabular}{lcccc}
\hline & \multicolumn{4}{c}{$\mathrm{Q}_{1}$} \\
$\operatorname{Pn}(N)$ & $\mathrm{Fc}^{\mathrm{opt}}$ & $T$ & $V\left(V_{\text {int }}\right)$ & $C$ \\
& 2638270 & 234 & $1708(1664)$ & 1399 \\
& 3046250 & 298 & $2092(2045)$ & 1399 \\
$\operatorname{Pn}_{15}(50)$ & 311 & $2252(2208)$ & 1399 \\
$\operatorname{Pn}_{16}(60)$ & 3970820 & 311 & 1399 \\
$\operatorname{Pn}_{17}(70)$ & 4854680 & 345 & $2524(2480)$ & 1398 \\
$\operatorname{Pn}_{18}(80)$ & 6034960 & 401 & $2791(2748)$ & 1398 \\
$\operatorname{Pn}_{19}(90)$ & 7475980 & 453 & $2951(2908)$ & \\
$\operatorname{Pn}_{20}(100)$ & & & &
\end{tabular}

(d)

\begin{tabular}{lcccc}
\hline & \multicolumn{4}{c}{$\mathrm{Q}_{2}$} \\
$\mathrm{PH}(N)$ & $\mathrm{Fc}^{\mathrm{opt}}$ & $T$ & $V\left(V_{\text {int }}\right)$ & $C$ \\
& 165180 & 11 & $821(792)$ & 1371 \\
& 570715 & 38 & $991(952)$ & 1381 \\
$\operatorname{Pn}_{11}(10)$ & 897015 & 74 & $1268(1224)$ & 1386 \\
$\operatorname{Pn}_{12}(20)$ & 1564990 & 151 & $1540(1496)$ & 1386 \\
$\operatorname{Pn}_{13}(30)$ & 2631090 & 204 & $1700(1656)$ & 1386 \\
$\operatorname{Pn}_{14}(40)$ & 2998950 & 260 & $2084(2040)$ & 1386 \\
$\operatorname{Pn}_{15}(50)$ & 3945430 & 296 & $2244(2200)$ & 1386 \\
$\operatorname{Pn}_{16}(60)$ & 4796250 & 321 & $2516(2472)$ & 1386 \\
$\operatorname{Pn}_{17}(70)$ & 6004750 & 378 & $2783(2740)$ & 1385 \\
$\operatorname{Pn}_{18}(80)$ & 7652490 & 421 & $2943(2900)$ & 1385 \\
$\operatorname{Pn}_{19}(90)$ & & & & \\
$\operatorname{Pn}_{20}(100)$ & & &
\end{tabular}

(e)

\begin{tabular}{|c|c|c|c|c|}
\hline \multirow[t]{2}{*}{$\operatorname{Pn}(N)$} & \multicolumn{4}{|c|}{$\begin{array}{c}\mathrm{Q}_{3} \\
\mathrm{DHIP} \\
\mathrm{HM}\end{array}$} \\
\hline & $\mathrm{Fc}^{\mathrm{opt}}$ & $T$ & $V\left(V_{\text {int }}\right)$ & C \\
\hline $\mathrm{Pn}_{11}(10)$ & 165180 & 9 & $801(772)$ & 1266 \\
\hline $\operatorname{Pn}_{12}(20)$ & 570715 & 31 & $971(922)$ & 1276 \\
\hline $\operatorname{Pn}_{13}(30)$ & 897015 & 67 & 1240 (1196) & 1381 \\
\hline $\operatorname{Pn}_{14}(40)$ & 1571600 & 134 & $1504(1460)$ & 1381 \\
\hline $\mathrm{Pn}_{15}(50)$ & 2634620 & 198 & $1664(1620)$ & 1381 \\
\hline $\operatorname{Pn}_{16}(60)$ & 3000310 & 234 & 2032 (1988) & 1381 \\
\hline $\mathrm{Pn}_{17}(70)$ & 4027740 & 287 & $2192(2148)$ & 1381 \\
\hline $\mathrm{Pn}_{18}(80)$ & 4916740 & 311 & $2456(2412)$ & 1381 \\
\hline $\mathrm{Pn}_{19}(90)$ & 5978220 & 318 & $2715(2672)$ & 1380 \\
\hline $\mathrm{Pn}_{20}(100)$ & 7475980 & 332 & 2875 (2832) & 1380 \\
\hline
\end{tabular}

$\mathrm{Fc}^{\mathrm{opt}}$ : the optimal value of $\mathrm{Fc}$ (objective function).

$T$ : time for finding solution (in seconds).

${ }^{*}$ The feasible value of the objective function after the time $T$.

${ }^{* *}$ Calculation was stopped after $600 \mathrm{~s}$.

$V\left(V_{\mathrm{int}}\right)$ : the number of decision variables (integer decision variables). $C$ : the number of constraints.

MILP: the implementation in the MP-based environment - MILP model. MILP_T: the implementation in the declarative hybrid implementation framework (DHIF) MILP model after transformation.

HM: implementation in the declarative hybrid implementation framework (DHIF) model after transformation.

\section{Conclusions}

In the highly competitive environment we are often forced to make quick and optimal decisions while assessing their costs.
TABLE 9: Analysis of the impact parameter $V_{s}$ (distributor maximum capacity) for $\mathrm{Fc}^{\mathrm{opt}}$.

\begin{tabular}{lccccc}
\hline \multirow{2}{*}{$=V_{1}=V_{2}=V_{3}=V_{4}$} & $\mathrm{Fc}^{\mathrm{opt}}$ & \multicolumn{4}{c}{ Distributor capacity } \\
& & $V_{x_{1}}$ & $V_{x_{2}}$ & $V_{x_{3}}$ & $V_{x_{4}}$ \\
\hline 500 & NFSF & - & - & - & - \\
600 & 1543390 & 550 & 598 & 600 & 297 \\
700 & 1165630 & 664 & 700 & 681 & 0 \\
800 & 1103310 & 510 & 760 & 775 & 0 \\
1000 & 991305 & 200 & 860 & 985 & 0 \\
1100 & 915015 & 0 & 975 & 1070 & 0 \\
1500 & 897015 & 0 & 925 & 1120 & 0 \\
2000 & 897015 & 0 & 925 & 1120 & 0 \\
2200 & 897015 & 0 & 925 & 1120 & 0 \\
2600 & 897015 & 0 & 925 & 1120 & 0 \\
3000 & 897015 & 0 & 925 & 1120 & 0 \\
\hline
\end{tabular}

NFSF: no feasible solution found.

TABLE 10: Analysis of the impact parameter $Z_{\mathrm{t}_{e}}$ (the number of transport units using mode of transport $e$ ) for $\mathrm{Fc}^{\text {opt }}$.

\begin{tabular}{lccccc}
\hline & \multicolumn{5}{c}{ Number of transport } \\
$Z_{\mathrm{t}_{1}}=Z_{\mathrm{t}_{2}}=Z_{\mathrm{t}_{3}}=Z_{\mathrm{t}_{4}}$ & $\mathrm{Fc}^{\mathrm{opt}}$ & \multicolumn{4}{c}{ units used } \\
& & $Z_{x_{1}}$ & $Z_{x_{2}}$ & $Z_{x_{3}}$ & $Z_{x_{4}}$ \\
\hline 10 & NFSF & - & - & - & - \\
15 & 1010170 & 5 & 3 & 14 & 15 \\
20 & 963165 & 5 & 3 & 12 & 20 \\
25 & 927915 & 5 & 3 & 9 & 25 \\
30 & 897015 & 5 & 3 & 7 & 29 \\
35 & 897015 & 5 & 3 & 7 & 29 \\
\hline
\end{tabular}

As it has been shown to support such decisions use of the presented approach and model is very effective.

The effectiveness of the proposed declarative hybrid method with multidimensional transformation is based on the reduction of a combinatorial optimization problem.

Thus, the application of this method practically to all the SCM optimization models ensures that the same or better solutions are found eight to more than one hundred times faster (very often optimal instead of feasible solutions). It needs to be highlighted that the proposed method not only enables faster solving of optimization problems but also enables faster solving of problems larger than those in $[22,25]$ (Table 8).

The proposed models and methods are highly recommended for all types of optimization problems in SCM or for similar problems where constraints include many discrete decision variables and their summation. Moreover, the proposed method allows successful modeling and solving of problems with logical constraints and therefore the DHIF implementations can be applied to area of logistics, transport, production, and scheduling or project management. 


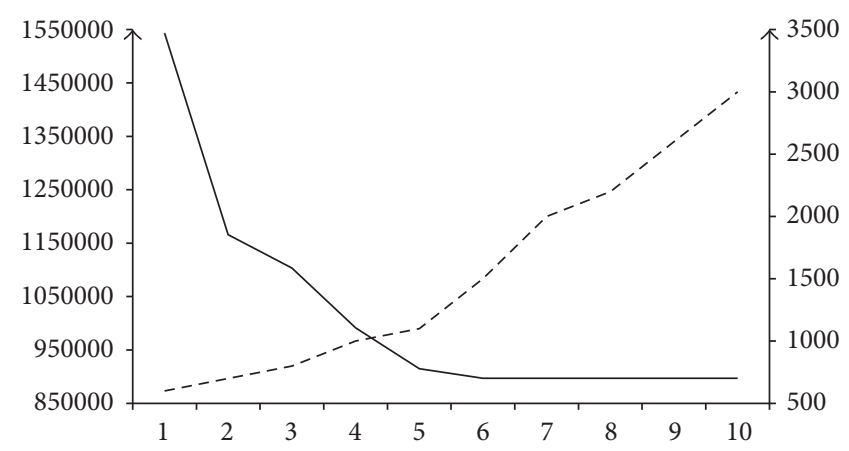
$-\mathrm{Fc}$
$--\mathrm{V}$

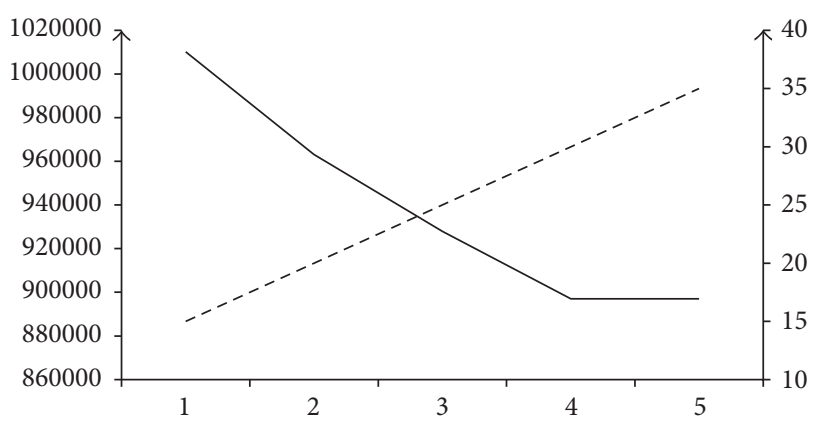

$-\mathrm{Fc}$

(a)

(b)

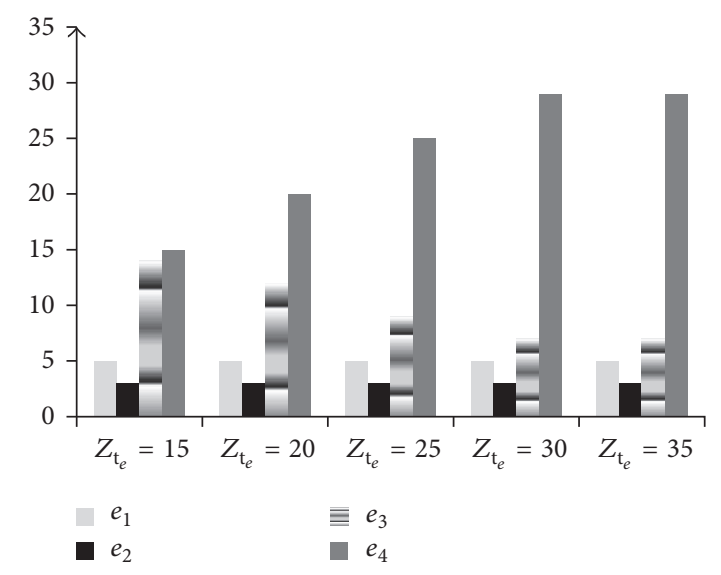

(c)

FIGURE 4: (a) Diagram visualizing the impact of parameter $V_{s}$ (distributor maximum capacity) for Fc ${ }^{\text {opt }}$. (b) Diagram visualizing the impact of parameter $Z_{\mathrm{t}_{e}}$ (number of transportation units using transportation mode $e$ ) for $\mathrm{Fc}^{\mathrm{opt}}$. (c) The number of used transport units (mode $e_{i}$, the limit $Z_{\mathrm{t}_{e}}$ ).

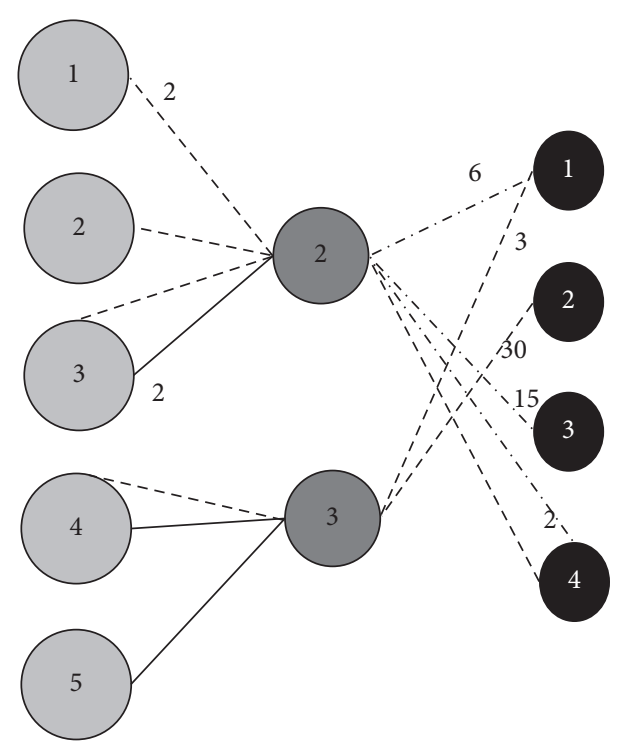

(a)

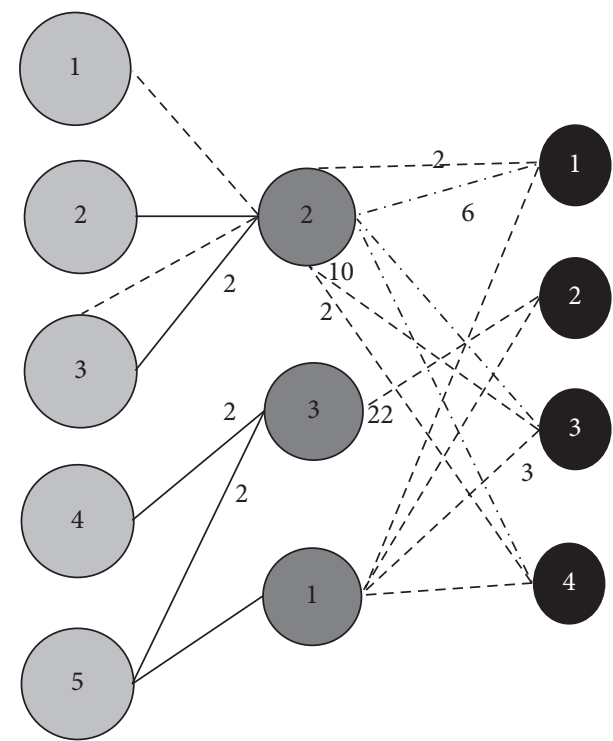

(b)

Figure 5: (a) The structure of the supply chain network with multimodal transport for $\mathrm{Pn}_{4}(40)$ and $\mathrm{Fc}^{\text {opt }}$. (b) The structure of the supply chain network with multimodal transport for $\mathrm{Pn}_{14}(40)$ with $\mathrm{Q}_{1}$ and $\mathrm{Fc}^{\mathrm{opt}}$. 
TABLE 11: The results of numerical examples for MILP_T with hard (9T) and soft (2S) constraints.

\begin{tabular}{|c|c|c|c|c|c|c|c|}
\hline $\mathrm{P}_{i}(N)$ & $e$ & $Z_{\mathrm{t}_{e}}$ & $V$ & C & $T$ & $\mathrm{Fc}^{\mathrm{opt}}$ & $Z_{x_{e}}$ \\
\hline \multicolumn{8}{|c|}{ Hard } \\
\hline \multirow{4}{*}{$P_{1}(10)$} & 1 & 2 & \multirow{4}{*}{846 (818) } & \multirow{4}{*}{1385} & \multirow{4}{*}{7} & \multirow{4}{*}{619} & 1 \\
\hline & 2 & 2 & & & & & 1 \\
\hline & 3 & 3 & & & & & 3 \\
\hline & 4 & 4 & & & & & 4 \\
\hline \multirow{4}{*}{$\mathrm{P}_{2}(20)$} & 1 & 2 & \multirow{4}{*}{1015 (978) } & \multirow{4}{*}{1395} & \multirow{4}{*}{121} & \multirow{4}{*}{1584} & 2 \\
\hline & 2 & 5 & & & & & 5 \\
\hline & 3 & 10 & & & & & 7 \\
\hline & 4 & 15 & & & & & 15 \\
\hline \multirow{4}{*}{$\mathrm{P}_{3}(30)$} & 1 & 3 & \multirow{4}{*}{$1292(1250)$} & \multirow{4}{*}{1400} & \multirow{4}{*}{-} & \multirow{4}{*}{ NFFS } & - \\
\hline & 2 & 4 & & & & & - \\
\hline & 3 & 10 & & & & & - \\
\hline & 4 & 15 & & & & & - \\
\hline \multirow{4}{*}{$\mathrm{P}_{4}(40)$} & 1 & 3 & \multirow{4}{*}{1564 (1522) } & \multirow{4}{*}{1400} & & & 3 \\
\hline & 2 & 10 & & & 159 & 3675 & 10 \\
\hline & 3 & 15 & & & & & 14 \\
\hline & 4 & 30 & & & & & 30 \\
\hline & & Sot & $K_{\mathrm{rd}_{1}}=K_{\mathrm{rd}_{2}}=$ & $\mathrm{rrd}_{3}=1$ & $t_{4}=1$ & & \\
\hline & 1 & 2 & & & & & 1 \\
\hline$P$ & 2 & 2 & 851 (822) & 1386 & 6 & 603 & 1 \\
\hline$P_{1}(10)$ & 3 & 3 & & 1000 & 0 & 000 & 1 \\
\hline & 4 & 4 & & & & & 8 \\
\hline & 1 & 2 & & & & & 3 \\
\hline $\mathrm{P}_{2}(20)$ & 2 & 5 & $1020(982)$ & 1396 & 146 & 1568 & 3 \\
\hline & 3 & 10 & & & 170 & & 16 \\
\hline & 4 & 15 & & & & & 17 \\
\hline & 1 & 3 & & & & & 4 \\
\hline $\mathrm{P}_{3}(30)$ & 2 & 4 & $1297(1254)$ & 1401 & 510 & 2461 & 5 \\
\hline & 3 & 10 & & & & & 7 \\
\hline & 4 & 15 & & & & & 29 \\
\hline & 1 & 3 & & & & & 6 \\
\hline $\mathrm{P}_{4}(40)$ & 2 & 10 & 1569 (1526) & 1401 & 89 & 3599 & 4 \\
\hline $14(\mathrm{TO})$ & 3 & 15 & & & & & 9 \\
\hline & 4 & 30 & & & & & 40 \\
\hline & & Sot & $K_{\mathrm{rd}_{1}}=K_{\mathrm{rd}_{2}}=$ & $\mathrm{rd}_{3}=1$ & $H_{4}=5$ & & \\
\hline & 1 & 2 & & & & & 1 \\
\hline$P_{1}(10)$ & 2 & 2 & 851 (822) & 1386 & 5 & 619 & 1 \\
\hline $11(10)$ & 3 & 3 & $001(022)$ & 1000 & J & (3) & 1 \\
\hline & 4 & 4 & & & & & 8 \\
\hline & 1 & 2 & & & & & 3 \\
\hline$P_{2}(20)$ & 2 & 5 & 1020 (982) & 1396 & 227 & 1580 & 3 \\
\hline$I_{2}(20)$ & 3 & 10 & $1020(702)$ & 1070 & 221 & 1000 & 16 \\
\hline & 4 & 15 & & & & & 17 \\
\hline & 1 & 3 & & & & & 4 \\
\hline $\mathrm{P}_{3}(30)$ & 2 & 4 & $1297(1254)$ & 1401 & 203 & 2560 & 4 \\
\hline & 3 & 10 & & & & & 10 \\
\hline & 4 & 15 & & & & & 23 \\
\hline & 1 & 3 & & & & & 6 \\
\hline $\mathrm{P}_{4}(40)$ & 2 & 10 & 1569 (1526) & 1401 & 263 & 3651 & 4 \\
\hline & 3 & 15 & & & & & 9 \\
\hline & 4 & 30 & & & & & 40 \\
\hline
\end{tabular}

TABLE 11: Continued.

\begin{tabular}{|c|c|c|c|c|c|c|c|}
\hline $\mathrm{P}_{i}(N)$ & $e$ & $Z_{\mathrm{t}_{e}}$ & V & C & $T$ & $\mathrm{Fc}^{\mathrm{opt}}$ & $Z_{x_{e}}$ \\
\hline \multicolumn{8}{|c|}{ Soft $K_{\mathrm{rd}_{1}}=K_{\mathrm{rd}_{2}}=K_{\mathrm{rd}_{3}}=K_{\mathrm{rd}_{4}}=10$} \\
\hline \multirow{4}{*}{$\mathrm{P}_{1}(10)$} & 1 & 2 & \multirow{4}{*}{$851(822)$} & \multirow{4}{*}{1386} & \multirow{4}{*}{8} & \multirow{4}{*}{619} & 1 \\
\hline & 2 & 2 & & & & & 1 \\
\hline & 3 & 3 & & & & & 3 \\
\hline & 4 & 4 & & & & & 4 \\
\hline \multirow{4}{*}{$\mathrm{P}_{2}(20)$} & 1 & 2 & \multirow{4}{*}{$1020(982)$} & \multirow{4}{*}{1396} & \multirow{4}{*}{185} & \multirow{4}{*}{1584} & 2 \\
\hline & 2 & 5 & & & & & 5 \\
\hline & 3 & 10 & & & & & 7 \\
\hline & 4 & 15 & & & & & 15 \\
\hline \multirow{4}{*}{$\mathrm{P}_{3}(30)$} & 1 & 3 & \multirow{4}{*}{$1297(1254)$} & \multirow{4}{*}{1401} & \multirow{4}{*}{383} & \multirow{4}{*}{2616} & 4 \\
\hline & 2 & 4 & & & & & 4 \\
\hline & 3 & 10 & & & & & 11 \\
\hline & 4 & 15 & & & & & 21 \\
\hline \multirow{4}{*}{$\mathrm{P}_{4}(40)$} & 1 & 3 & \multirow{4}{*}{1569 (1526) } & \multirow{4}{*}{1401} & \multirow{4}{*}{318} & \multirow{4}{*}{3668} & 6 \\
\hline & 2 & 10 & & & & & 4 \\
\hline & 3 & 15 & & & & & 14 \\
\hline & 4 & 30 & & & & & 30 \\
\hline
\end{tabular}

$e$ : mode of transport $(e=1 \cdots E)$.

$Z_{\mathrm{t}_{e}}:$ number of transportation units using transportation mode $e$.

$Z_{x_{e}}$ : number of used transportation units using transportation mode $e$.

$K_{\mathrm{rd}_{i}}$ : penalty coefficient.

NFFS: Not found feasible solution.

In addition to nonquestionable effectiveness and efficiency of the proposed method, it offers unlimited possibilities of optimization problem modeling. This method is also applicable to problems with soft constraints (Table 11).

The possibility of optimization models, which also have soft and logical constraints, is very important for practical applications in the highly competitive environment (in case of violation certain constraints and assessing the impact on the objective function).

Future studies of the proposed method and framework will focus on the following: (a) modeling problems with other logical and nonlinear constraints and so forth, (b) multiobjective optimization, and (c) introduction of fuzzy logic [26] and uncertainty to the models.

\section{Competing Interests}

The authors declare that there is no conflict of interests regarding the publication of this paper.

\section{References}

[1] B. M. Beamon, "Supply chain design and analysis: models and methods," International Journal of Production Economics, vol. 55, no. 3, pp. 281-294, 1998.

[2] G. Q. Huang, J. S. K. Lau, and K. L. Mak, "The impacts of sharing production information on supply chain dynamics: a review of the literature," International Journal of Production Research, vol. 41, no. 7, pp. 1483-1517, 2003.

[3] J. Mula, D. Peidro, M. Díaz-Madroñero, and E. Vicens, "Mathematical programming models for supply chain production and transport planning," European Journal of Operational Research, vol. 204, no. 3, pp. 377-390, 2010. 
[4] K. Apt and M. Wallace, Constraint Logic Programming Using Eclipse, Cambridge University Press, New York, NY, USA, 2006.

[5] P. Sitek and J. Wikarek, "A hybrid programming framework for modeling and solving constraint satisfaction and optimization problems," Scientific Programming, vol. 2016, Article ID 5102616, 13 pages, 2016.

[6] F. Rossi, P. Van Beek, and T. Walsh, Handbook of Constraint Programming (Foundations of Artificial Intelligence), Elsevier Science, New York, NY, USA, 2006.

[7] G. Bocewicz and Z. A. Banaszak, "Declarative approach to cyclic steady state space refinement: periodic process scheduling," International Journal of Advanced Manufacturing Technology, vol. 67, no. 1-4, pp. 137-155, 2013.

[8] K. Bzdyra, G. Bocewicz, and Z. Banaszak, "Mass customized projects portfolio scheduling-imprecise operations time approach," Applied Mechanics and Materials, vol. 791, pp. 70-80, 2015.

[9] M. G. Buscemi and U. Montanari, "A survey of constraint-based programming paradigms," Computer Science Review, vol. 2, no. 3, pp. 137-141, 2008.

[10] G. Kondrak and P. van Beek, "A theoretical evaluation of selected backtracking algorithms," Artificial Intelligence, vol. 89, no. 1-2, pp. 365-387, 1997.

[11] S. Bistarelli, U. Montanari, and F. Rossi, "Semiring-based constraint satisfaction and optimization," Journal of the ACM, vol. 44, no. 2, pp. 201-236, 1997.

[12] M. Milano and M. Wallace, "Integrating operations research in constraint programming," Annals of Operations Research, vol. 175, pp. 37-76, 2010.

[13] T. Achterberg, T. Berthold, T. Koch, and K. Wolter, "Constraint integer programming. A new approach to integrate CP and MIP," in Integration of AI and OR Techniques in Constraint Programming for Combinatorial Optimization Problems, vol. 5015 of Lecture Notes in Computer Science, pp. 6-20, Springer, 2008.

[14] P. Sitek and J. Wikarek, "A hybrid method for modeling and solving constrained search problems," in Proceedings of the Federated Conference on Computer Science and Information Systems (FedCSIS '13), pp. 385-392, IEEE, Kraków, Poland, September 2013.

[15] P. Sitek and J. Wikarek, "Hybrid solution framework for supply chain problems," in Distributed Computing and Artificial Intelligence, 11th International Conference, S. Omatu, H. Bersini, J. M. Corchado, S. Rodríguez, P. Pawlewski, and E. Bucciarelli, Eds., vol. 290 of Advances in Intelligent Systems and Computing, pp. 11-18, Springer, Berlin, Germany, 2014.

[16] J. N. Hooker, "Logic, optimization, and constraint programming," INFORMS Journal on Computing, vol. 14, no. 4, pp. 295321, 2002.

[17] V. Jain and I. E. Grossmann, "Algorithms for hybrid MILP/CP models for a class of optimization problems," INFORMS Journal on Computing, vol. 13, no. 4, pp. 258-276, 2001.

[18] Eclipse, "Eclipse-The Eclipse Foundation open source community website," 2015, https://eclipse.org/.

[19] K. Shen and J. Schimpf, "Eplex: harnessing mathematical programming solvers for constraint logic programming," in Principles and Practice of Constraint Programming-CP 2005, vol. 3709 of Lecture Notes in Computer Science, pp. 622-636, Springer, Berlin, Germany, 2005.
[20] A. Bockmayr and T. Kasper, "Branch-and-infer: a framework for combining CP and IP," in Constraint and Integer Programming, vol. 27 of Operations Research/Computer Science Interfaces Series, pp. 59-87, Springer, 2004.

[21] Q. Dang, I. E. Nielsen, and K. Steger-Jensen, "Scheduling a single mobile robot incorporated into production environment," in EcoProduction and Logistics-Emerging Trends and Business Practices, part 2, P. Golinska, Ed., EcoProduction, pp. 185-201, Springer, Berlin, Germany, 2013.

[22] P. Sitek and J. Wikarek, "Cost optimization of supply chain with multimodal transport," in Proceedings of the Federated Conference on Computer Science and Information Systems (FedCSIS '12), pp. 1111-1118, IEEE, Wroclaw, Poland, September 2012.

[23] A. Schrijver, Theory of Linear and Integer Programming, John Wiley \& Sons, New York, NY, USA, 1998.

[24] K. Grzybowska, "Sustainability in the supply chain: analysing the enablers," in Environmental Issues in Supply Chain Management: New Trends and Applications, P. Golinska and C. A. Romano, Eds., EcoProduction, pp. 25-40, Springer, Berlin, Germany, 2012.

[25] P. Sitek and J. Wikarek, "A hybrid framework for the modelling and optimisation of decision problems in sustainable supply chain management," International Journal of Production Research, vol. 53, no. 21, pp. 6611-6628, 2015.

[26] M. Relich and W. Muszynski, "The use of intelligent systems for planning and scheduling of product development projects," Procedia Computer Science, vol. 35, pp. 1586-1595, 2014. 


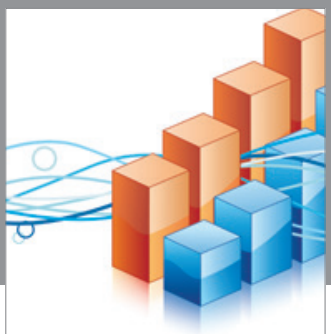

Advances in

Operations Research

vatem alat4

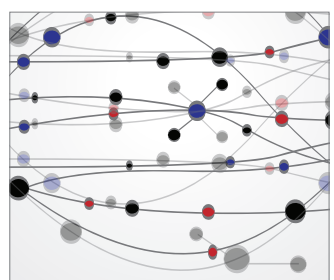

\section{The Scientific} World Journal
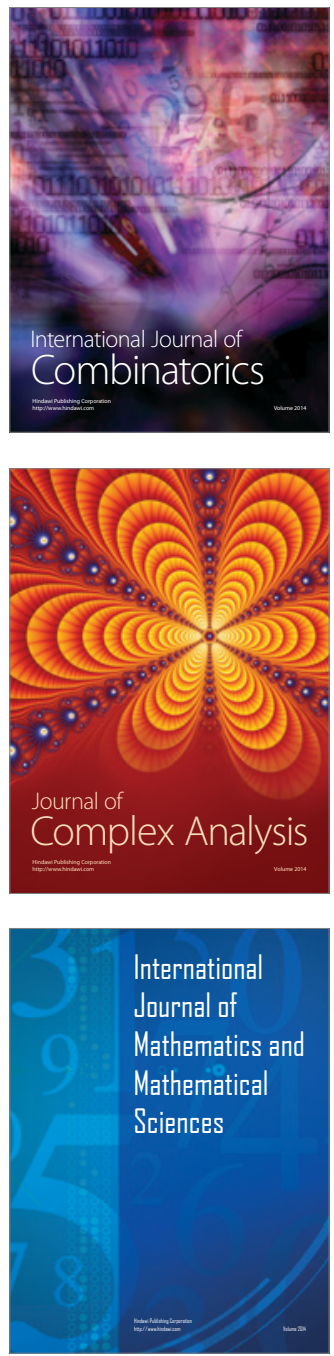
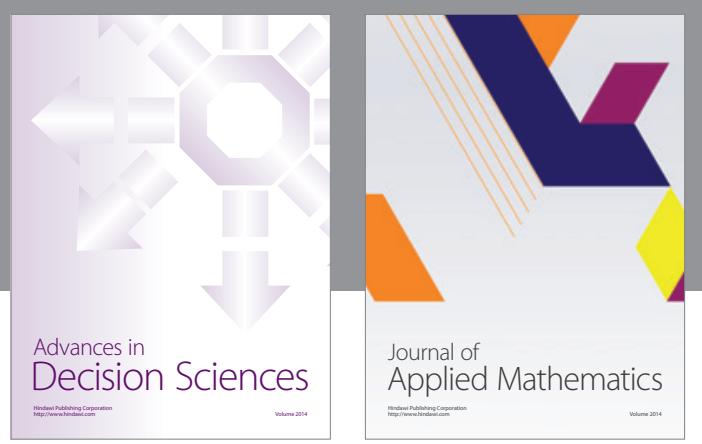

Algebra

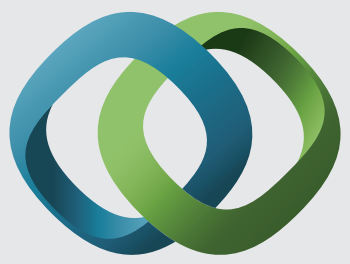

\section{Hindawi}

Submit your manuscripts at

http://www.hindawi.com
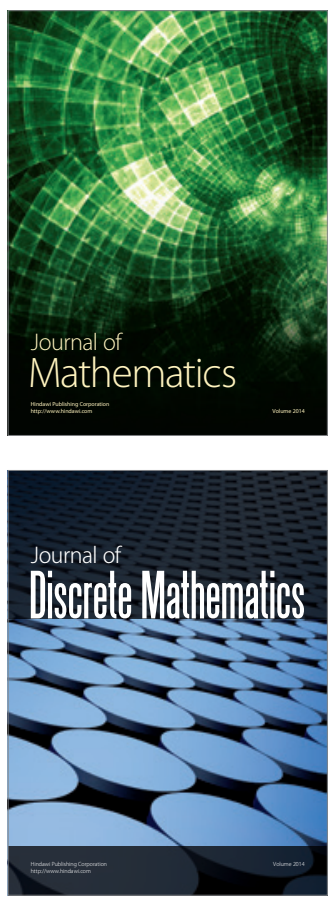

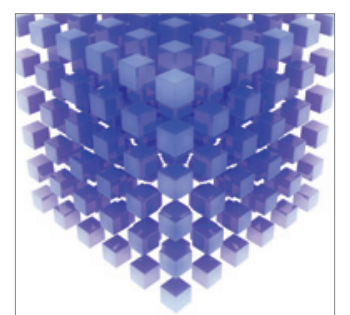

Mathematical Problems in Engineering
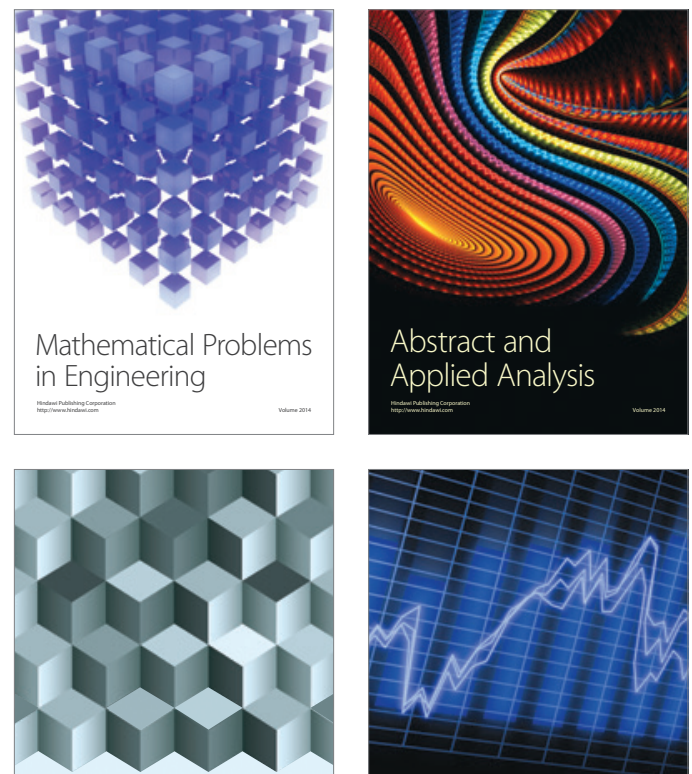

Journal of

Function Spaces

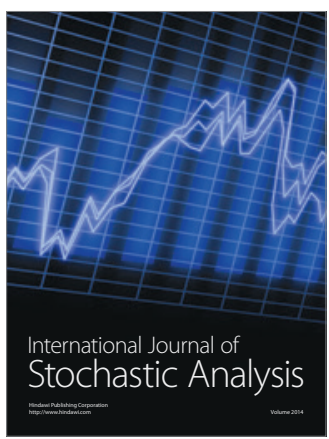

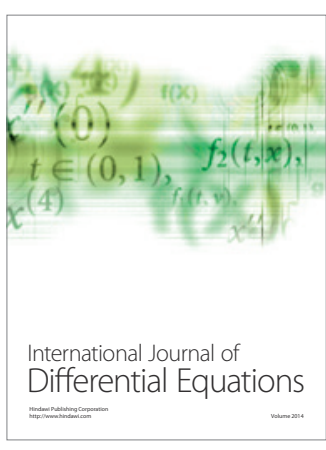
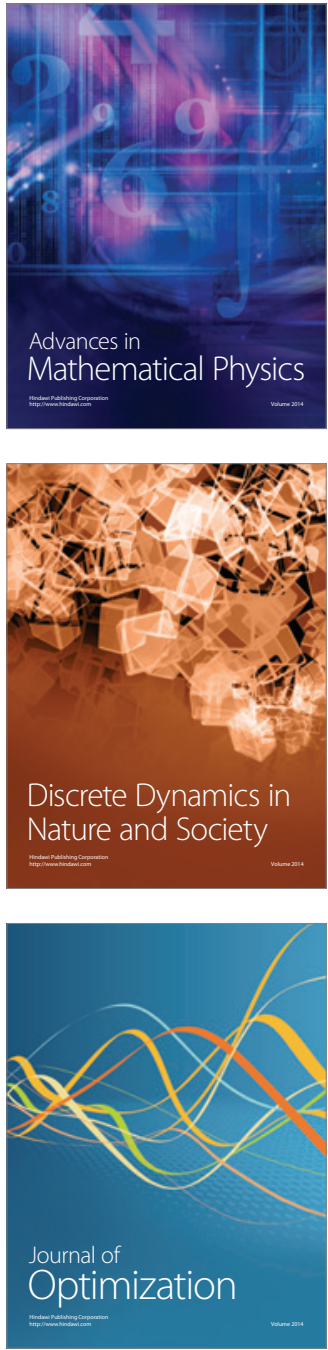